Pacific Journal of Mathematics

A CHARACTERIZATION, EXISTENCE PROOF AND
DIMENSION BOUNDS FOR THE KERNEL OF A GAME 


\title{
A CHARACTERIZATION, EXISTENCE PROOF AND DIMENSION BOUNDS FOR THE KERNEL OF A GAME
}

\author{
M. Maschler and B. Peleg
}

The kernel of a cooperative game is a subset of the bargaining set $\mathscr{C}_{1}^{(i)}$. It is sensitive to symmetry relations and their generalizations, which may exist in the characteristic function. The present paper offers an interesting representation formula for the kernel. This formula is applied to deriving properties of the kernel as well as practical methods for its computation.

In particular, we provide an algebraic proof to the theorem stating that for each coalition structure in a cooperative game there exists a payoff in the kernel (and therefore also in the bargaining set $\mathscr{M}_{1}^{(i)}$ ). (All other known proofs of this theorem are based on the Brouwer fixed-point theorem.) We also prove that the maximal dimension of the kernel of an $n$-person game is $n-\left[\log _{2}\left(n-\frac{1}{2}\right)\right]-2$, and this bound is sharp.

Two players in a game are called symmetric, if the game remains invariant when these players exchange roles. One generalizes this concept by defining a player $k$ to be more desirable than a player $l$, if player $k$ always contributes not less than player $l$ by joining coalitions which contain none of these players. It turns out that the payoffs in the kernel always preserve the order determined by the desirability relations. This fact may simplify the representation formula significantly.

Introduction and general background. Let $G$ be an $n$-person cooperative game with side payments, and let it be known that its participants are trying to form a certain coalition-structure. How could or should each coalition divide its proceeds among its members? Attempting to answer this question by imposing certain stability requirements on the outcomes, R. J. Aumann and M. Maschler have introduced in [1] various bargaining sets, the most important of which is the bargaining set $\mathscr{C l}_{1}^{(i)}$. (This particular set is especially treated in M. Davis and M. Maschler [3] and in B. Peleg [9].)

The study of some existence theorems for $\mathscr{M}_{1}^{(i)}$ led M. Davis and M. Maschler [4] to construct a subset $\mathscr{K}$ of this bargaining set, called the kernel of the game $G$. Being a subset of $\mathscr{M}_{1}^{(i)}$, each outcome in the kernel possesses the same stability properties which characterize

Received August 11, 1964, and in revised Form January 8, 1965. The research described in this paper was partially supported by the U.S. Office of Naval Research, Logistics and Mathematical Statistics Branch, under Contract Number N62558-3586. 
the outcomes of $\mathscr{C}_{1}^{(i)}$. Moreover, it turns out that the kernel possesses many interesting mathematical properties, especially reflecting many symmetries that may exist in $G$. It seems that it represents a certain central portion of the bargaining set, though this aspect deserves further study. From the intuitive point of view, however, the arguments that the players will prefer outcomes in the kernel to other outcomes in the bargaining set are not too convincing (see [4]). At most, the kernel represents a very restrictive type of negotiation. However, if the units of the payoffs represent interpersonally compared units of utility, the kernel also represents payoffs which reflect a certain balance of power among the members. (See the definition in $§ 2$.) (See also [7], [8].)

Being what it is, the kernel is easier to handle than the bargaining set and it is more amenable to computations. As a matter of fact, this paper has been stimulated by the results of computing the kernel for all the 4 and 5-person weighted majority games, work conducted by R. J. Aumann, B. Peleg and P. Rabinowitz [2].

Unlike other papers, in which the results were mainly obtained by a direct study of the inequalities which define the kernel and the various bargaining sets, the present paper stems from a new characterization of the kernel by a representation formula, based on separation relations induced by sets of coalitions $(\S 4)$. The separation relations are defined and studied in $\S 3$. They lead to interesting problems of algebraic and combinatorial nature which are discussed throughout the paper. In particular, it is shown how to construct sets of coalitions which possess certain separation relations ( $(8)$.

The representation formula describes the kernel as a finite union of closed and convex polyhedra. At first sight, the formula looks much more complicated than the original definition of the kernel; however, its great power is revealed in almost any subsequent section. For example, it yields a completely algebraic proof that for every game (and corresponding to every coalition structure) there exist payoffs in the kernel $(\S 5)$. This result has previously been proved by the use of the Brouwer fixed-point theorem (see [3], [4] and [9]). A by-product of the method of the proof is the quite surprising fact that the kernel always intersects the core of the game, if the core is not empty.

The study of the separation relations and the representation formula enables one to determine the maximal dimension of the kernel of an $n$-person game $(\S 6)$. It equals precisely $n-\left[\log _{2}\left(n-\frac{1}{2}\right)\right]-2$ (where, "[ ]" means, "the integral part of"). ${ }^{1}$ In addition to its theoretical interest, this result is important in devising a program for computing

1 The term $1 / 2$ is needed in order to make the formula correct also for 1-person games. 
the kernel (see [2] $\S 6$ ).

There is an enormous number of polyhedra which appear in the representation formula. Experience in computation shows, however, that most of them are either empty sets or are contained in a few of them. For computation purposes, one is interested in screening out a priori those polyhedra which are superfluous. The problem is two-fold:

(1) To determine which polyhedra are superfluous for all games $(\S 7)$.

(2) To determine which polyhedra are superfluous due to the fact that the characteristic function happens to possess certain properties ( $\S \S 10$ and 11).

For example, two players in a game are called symmetric if the game remains invariant when these players exchange roles. We generalize this concept by defining a player $k$ to be more desirable than a player $l$, if player $k$ always contributes not less than player $l$ by joining coalitions which contain none of these players. These desirability relations were studied by J. R. Isbell [6] in the case of simple games. It turns out that the payoffs in the kernel always preserve the order determined by the desirability relations. If many of them exist, one is able to reduce the representation formula quite significantly $(\S 10)$.

Three examples are provided in $\S 10$, in order to illustrate how to use the theoretical results for actual computation of the kernel of a game.

A similar, but essentially different, method of reduction is presented in $\S 11$.

2. Notation and basic definitions. We shall be concerned with an $n$-person cooperative game $G=(N ; v)$, with side payments, described by the set $N=\{1,2, \cdots, n\}$ of $n$ players and the characteristic function $v=v(S)$, which maps the nonempty subsets of $N$, called coalitions, into the real numbers. $v(S)$ will be called the value of the coalition $S$, and the characteristic function will be normalized by the requirement. ${ }^{2}$

$$
v(i)=0, \quad i=1,2, \cdots, n .
$$

We do not require $v(S)$ to be super-additive, but in order to avoid trivial restrictions on the coalition structures, we shall assume

$$
v(S) \geqq 0 \text { for each coalition } S \text { (see (2.4)). }
$$

${ }^{2}$ For simplicity, commas and brackets will be omitted whenever there is no question of ambiguity. Thus, $v(i)$ will be written instead of $v(\{i\})$, and $v(12)$ will mean $v(\{1,2\})$ and not $v(\{12\})$. 
When such a game is being played, presumably the players partition themselves into coalitions, and each coalition formed distributes its value among its members. It is reasonable to assume that each player receives at least the amount he can get by playing as a 1person coalition (individual rationality). Thus, an outcome of the game can be represented by an individually rational payoff configuration (i.r.p.c.)

$$
(x ; \mathscr{B})=\left(x_{1}, x_{2}, \cdots, x_{n} ; B_{1}, B_{2}, \cdots, B_{m}\right) .
$$

Here, $\mathscr{B}=\left\{B_{1}, B_{2}, \cdots, B_{m}\right\}$ is a partition of $N$ into coalitions and it is called the coalition structure (c.s). $x=\left(x_{1}, x_{2}, \cdots, x_{n}\right)$ - the payoff vector (p.v.) - is a point in the cartesian product of $m$ simplexes $X(\mathscr{B})=X^{B_{1}} \times X^{B_{2}} \times \cdots \times X^{B_{m}}$, where

$$
X^{B_{j}}=\left\{x^{B_{j}}: \sum_{i \in B_{j}} x_{i}=v\left(B_{j}\right), x_{i} \geqq 0, i \in B_{j}\right\}, \quad j=1,2, \cdots, m,
$$

and $x^{B_{j}}$ is a $k_{j}$-tuple resulting from $x$ by considering only the coordinates indexed by the members of $B_{j}$, ( $k_{j}$ being the number of players in $\left.B_{j}\right)$.

In [4], Davis and Maschler introduced the concept of the kernel $\mathscr{K}=\mathscr{K}(G)$ of the game $G$ :

Let $(x ; \mathscr{B})$ be an i.r.p.c., and let $S$ be an arbitrary coalition (not necessarily in $\mathscr{B})$. We call

$$
e(S)=e(S, x)=v(S)-\sum_{i \in S} x_{i}
$$

the excess of $S$ with respect to $(x ; \mathscr{B})$. It represents the amount that $S$ can gain (lose, if $e(S)<0$ ), if its members leave $(x ; \mathscr{B})$ and form their own coalition.

Consider two players $k$ and $l$ who belong to the same coalition in $\mathscr{B}$. Denote by $\mathscr{T}_{k, l}$ the set of coalitions which contain player $k$ and do not contain player $l$; i.e.,

$$
\mathscr{T}_{k, l}=\{S: k \in S, l \notin S, S \subset N\} \text {. }
$$

The maximum excess of $k$ over $l$, with respect to $(x ; \mathscr{B})$ is defined to be

$$
s_{k, l}=s_{k, l}(x)=\max _{S \in \mathscr{T}_{k, l}} e(S) .
$$

We also say that player $k$ outweighs player $l$, with respect to $(x, \mathscr{B})$, and denote this by $k \gg l$, if $s_{k, l}>s_{l, k}$ and $x_{l}>0$. We say that two players $k$ and $l$ are in equilibrium with respect to $(x ; \mathscr{B})$, and denote this by $k \approx l$, if neither $k \gg l$ nor $l \gg k$, with respect to $(x ; \mathscr{B})$. Thus, 


$$
k \approx l \Leftrightarrow\left(s_{k, l}-s_{l, k}\right) x_{l} \leqq 0 \text { and }\left(s_{l, k}-s_{k, l}\right) x_{k} \leqq 0 .
$$

Two players who belong to different coalitions in $\mathscr{B}$ are always said to be in equilibrium.

Although $\gg$ is a transitive relation, it can be shown that $\approx$ may contain intransitivities. (See [4].)

A coalition $B_{j}, B_{j} \in \mathscr{B}$, is said to be balanced with respect to an i.r.p.c. $(x ; \mathscr{B})$, if each pair of players in $B_{j}$ is in equilibrium.

Definition 2.1. The kernel $\mathscr{K} \equiv \mathscr{K}(G)$ of a game $G$ is the set of all the i.r.p.c.'s having only balanced coalitions.

The set of all vectors $x$ such that $(x, \mathscr{B}) \in \mathscr{K}$ is clearly a finite union of closed convex polyhedra. A priori these may be enormous in number, even for games with 4 or 5 players; yet experience indicates that many of the systems of inequalities defining the polyhedra have no solution. In fact, in computing kernels for many 4 and 5-person games, we never came across more than 4 polyhedra composing the kernel.

One of the objects of this work will be to characterize these polyhedra and to analyze the structure of the kernel. We shall then use the results to give algebraic existence proofs, to establish an upper bound for the dimension of the kernel and to develop techniques for computing the kernel.

To simplify the exposition, we shall focus our attention on i.r.p.c.'s whose coalition-structure is the grand coalition $N$. Most results can easily be extended to other coalition structures, and the main ones will be stated without proofs. For this reason, except in Theorem 4.6, 6.7 and $9.3 \mathrm{a}$, the term payoff vector will henceforth denote an $n$-dimensional nonnegative vector, the sum of whose coordinates equals $v(N)$; i.e., a point in $X(N)$ (see (2.4)). Similarly, contrary to the general definition, we shall write $x \in \mathscr{K}$ if and only if $(x ; N) \in \mathscr{K}$.

3. Separation relations induced by a set of coalitions. Let $\mathscr{D}$ be a set of coalitions whose members belong to a set $N$ of players.

(i) We shall say that two players $k$ and $l$ separate each other by $\mathscr{D}$, or are separated by $\mathscr{D}$, if there exist coalitions $A$ and $B$ in $\mathscr{D}$ such that $k \in A, l \notin A, l \in B, k \notin B$; i.e.,

$$
k \in A \in \mathscr{D} \cap \mathscr{T}_{k, l} \text { and } l \in B \in \mathscr{D} \cap \mathscr{T}_{l, k} .
$$

(ii) We shall say that players $k$ and $l$ are inseparable by $\mathscr{D}$, if each coalition of $\mathscr{D}$ either contains both players or contains none of them. In other words,

$$
k \in A \in \mathscr{D} \text { if and only if } l \in A \in \mathscr{D} .
$$


(iii) We shall say that player $k$ separates player $l$ out by $\mathscr{D}$, if there exists a coalition $A$ in $\mathscr{D}$ such that $k \in A, l \notin A$, whereas every coalition in $\mathscr{D}$ which contains player $l$ contains also player $k$; i.e.,

$$
\begin{gathered}
k \in A \in \mathscr{D} \cap \mathscr{T}_{k, l}, \\
l \in B \in \mathscr{D} \text { implies } k \in B .
\end{gathered}
$$

Clearly, any two players either separate each other or are inseparable or one separates the other out and only one of these relations holds for them for a fixed $\mathscr{D}$.

The above separation concepts can be generated by a weak partial order relation $k \geqq{ }_{\mathscr{D}} l$ which means: every coalition in $\mathscr{D}$ which contains $l$ also contains $k$. With this notation, $k=l$ if and only if $k$ and $l$ are inseparable in $\mathscr{D}$. The relation $=_{\mathscr{D}}$ is an equivalence relation. Also, $k$ and $l$ separate each other if and only if they are incomparable by $\geqq_{\mathscr{D}}$; and $k>\mathscr{C} l$ if and only if $k$ separates $l$ out by $\mathscr{D}$.

We shall often be interested only in separation relations among players who belong to a subset $T$ of $N$, (allowing, of course, for the coalitions in $\mathscr{D}$ to contain also members of $N-T$ ). In this connection it makes a difference if a player $k$ who separates a player $l$ out, for $l \in T$, belongs or does not belong himself to $T$. Accordingly,

(iv) We shall say that a player $l$ in $T$ is separated out by $\mathscr{D}$, with respect to $T$, if $k \in T$ and $k$ separates $l$ out by $\mathscr{D}$.

Let $\mathscr{D}$ be a set of coalitions and let $T$ be a subset of $N$; then there exists a unique partition of $T$,

$$
T=T_{1} \cup T_{2} \cup \cdots \cup T_{u} \cup F(\mathscr{D}, T),
$$

which has the following properties:

(i) $F(\mathscr{D}, T)$ is the set of players who are separated out by $\mathscr{D}$, with respect to $T$.

(ii) $T_{1}, \cdots, T_{u}$ are the equivalence classes generated by the relation $=_{\mathscr{S}}$ on the players who are maximal in the relation $\geq_{\mathscr{D}}$.

We shall refer to $\left\{T_{1}, T_{2}, \cdots, T_{u}, F(\mathscr{D}, T)\right\}$ as the partition of $T$ induced by $\mathscr{D}$. The following is a direct consequence of the definitions:

Lemma 3.1. The partition $\left\{T_{1}, T_{2}, \cdots, T_{u}, F(\mathscr{D}, T)\right\}$, of $T$, induced by a set of coalitions $\mathscr{D}$ has the following properties:

(i) Two players in the same $T_{j}, j=1,2, \cdots, u$, are always inseparably by $\mathscr{D}$.

(ii) A player in $T_{i}$ and a player in $T_{j}, i \neq j$, are always separated by $\mathscr{D}$.

(iii) If $k \in T_{j}, j=1,2, \cdots, u$, and $l \in F(\mathscr{D}, T)$, then either $k$ separates $l$ out by $\mathscr{D}$, in which case every player of $T_{j}$ separates 
player $l$ out, or both players are separated by $\mathscr{D}$.

(iv) If $l \in F(\mathscr{D}, T)$, then there exists a player $k$ in a set $T_{j}$, $1 \leqq j \leqq u$, such that $k$ separates $l$ out by $\mathscr{D}$.

(v) If $\mathscr{D}$ contains a coalition which intersects but does not contain $T$, then, the partition contains at least two sets; otherwise, the partition is $T$ itself.

4. A representation of the kernel. Let $T$ be a subset of $N=\{1,2, \cdots, n\}$; we denote by $\mathscr{C}(T)$ the set of coalitions which intersect $T$ but do not contain $T$ :

$$
\mathscr{C}(T)=\bigcup_{k, l \in T} \mathscr{T}_{k, l}
$$

Let $x$ be a payoff vector in a game $(N ; v)$, we shall construct a profile of $x$ in the following way:

Denote by $q_{1}=\left\{T_{1}{ }^{1}\right\}$ the 1-element set, whose element $T_{1}{ }^{1}=N$ is the set of all the players. Denote by $\delta_{1}(x)=(\mathscr{D}(N, x))$ the vector having one component, where this component $\mathscr{D}(N, x)$ consists of all the coalitions in $\mathscr{C}(N)$ whose excess with respect to $x$ is maximal.

$$
\mathscr{D}(N, x)=\{S: S \in \mathscr{C}(N), e(S, x) \geqq e(T, x) \text { for all } T \in \mathscr{C}(N)\} .
$$

Denote by $s_{1}(x)$ this maximum excess; i.e.,

$$
s_{1}(x)=\operatorname{Max}_{S \in \mathscr{C}(N)} e(S, x) .
$$

The coalitions of $\mathscr{D}(N, x)$ induce the partition

$$
\left\{T_{1}^{2}, T_{2}^{2}, \cdots, T_{u_{2}}^{2}, F_{1}^{2}(\mathscr{D}(N, x), N)\right\}
$$

of the set $T_{1}^{1}$ (see (3.5)). Let $q_{2}=q_{2}(x)=\left\{T_{1}^{2}, T_{2}^{2}, \cdots, T_{u_{2}}^{2}\right\}$ be the set of the equivalence classes of the players in $N-F_{1}^{2}(\mathscr{D}(N, x), N)$. We continue inductively (see figure):

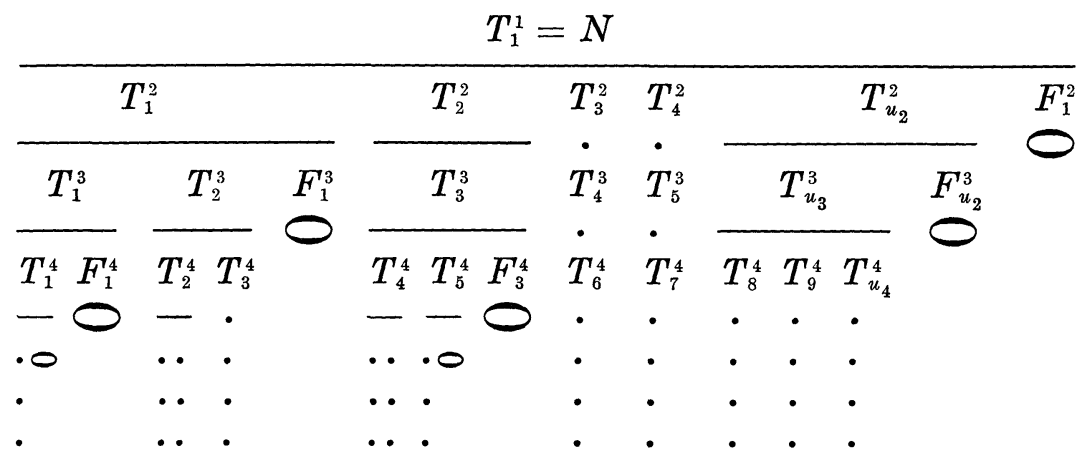

Suppose that $q_{r}=q_{r}(x)=\left\{T_{1}^{r}, T_{2}^{r}, \cdots, T_{u_{r}}^{r}\right\}$ has been defined. Consider

${ }^{3}$ Any one of the separation relations can hold for two players in $F(\mathscr{D}, T)$. 
the set $\mathscr{C}\left(q_{r}\right)=\mathscr{C}\left(T_{1}^{r}\right) \cup \mathscr{C}\left(T_{2}^{r}\right) \cup \cdots \cup \mathscr{C}\left(T_{u_{r}}^{r}\right)$, consisting of all the coalitions, each of which intersects, and does not contain, at least one of the sets $T_{j}^{r}, j=1,2, \cdots, u_{r}$. Among the coalitions in $\mathscr{C}\left(q_{r}\right)$ select the set $\mathscr{D}\left(q_{r}, x\right)$ consisting of those coalition in $\mathscr{C}\left(q_{r}\right)$ which attain a maximal excess; i.e.,

$$
\mathscr{D}\left(q_{r}, x\right)=\left\{S: S \in \mathscr{C}\left(q_{r}\right), e(S, x) \geqq e(T, x) \text { for all } T \in \mathscr{C}\left(q_{r}\right)\right\} .
$$

The coalitions in $\mathscr{D}\left(q_{r}, x\right)$ generate $u_{r}$ sets:

$$
\mathscr{D}\left(T_{j}^{r}, x\right)=\mathscr{D}\left(q_{r}, x\right) \cap \mathscr{C}\left(T_{j}^{r}\right), \quad j=1,2, \cdots, u_{r},
$$

consisting of those coalitions of maximum excess which happen to be in $\mathscr{C}\left(T_{j}^{r}\right), j=1,2, \cdots, u_{r}$. Denote by $\delta_{r}(x)=\left(\mathscr{D}\left(T_{1}^{r}, x\right), \mathscr{D}\left(T_{2}^{r}, x\right), \cdots\right.$, $\left.\mathscr{D}\left(T_{u_{r}}^{r}, x\right)\right)$ the $u_{r}$-tuple of these $\mathscr{D}\left(T_{j}^{r}, x\right)$ 's. It may well happen that some of its components are the same sets and that some are the empty set. The latter case will always occur e.g., if $T_{j}^{r}$ is a single player. However, if $\mathscr{C}\left(q_{r}\right)$ is not empty then $\mathscr{D}\left(q_{r}, x\right)$ contains at least one coalition. It $\mathscr{C}\left(q_{r}\right)$ is not empty, denote by $s_{r}(x)$ the excess of the coalitions in $\mathscr{D}\left(q_{r}, x\right)$; i.e.,

$$
s_{r}(x)=\operatorname{Max}_{S \in \mathscr{C}\left(q_{r}\right)} e(S, x) .
$$

Clearly

$$
s_{1}(x)>s_{2}(x)>\cdots,
$$

as long as the sequence is defined.

Each set $\mathscr{D}\left(T_{j}^{r}, x\right), j=1,2, \cdots, u_{r}$, induces a partition

$$
\left\{T_{j, 1}^{r+1}, T_{j, 2}^{r+1}, \cdots, T_{j, \nu_{j}}^{r+1}, F_{j}^{r+1}\left(\mathscr{D}\left(T_{j}^{r}, x\right), T_{j}^{r}\right)\right\}
$$

of $T_{j}^{r}$ (see (3.5)). Renumber all the equivalence classes in these partitions lexicographically in the lower indices and thus obtain the set $q_{r+1}=q_{r+1}(x)=\left\{T_{1}^{r+1}, T_{2}^{r+1}, \cdots, T_{u_{r+1}}^{r+1}\right\}$. Continue this process until you arrive at $q_{n}$. The set

$$
P=P(x)=\left\{q_{1} ; q_{2}, F_{1}^{2} ; q_{3}, F_{1}^{3}, \cdots, F_{u_{2}}^{3} ; \cdots ; q_{n}, F_{1}^{n}, \cdots, F_{u_{n-1}}^{n}\right\}
$$

is called the profile generated by $x$. Its subsets which are distinguished by semicolons are called its first stage, second stage, etc.

LEMma 4.1. Let $P$ be a profile generated by a payoff vector $x$ in a game $G$. The members of $q_{n}$ are single players.

Proof. If $\mathscr{C}\left(q_{r}\right)$ is not empty, then $\mathscr{D}\left(q_{r}, x\right)$ contains at least one coalition. Such a coalition belongs to at least one of the classes $\mathscr{C}\left(T_{j}^{r}\right), j=1,2, \cdots, u_{r}$. By part (v) of Lemma 3.1 the partition of $T_{j}^{r}$, 
induced by $\mathscr{D}\left(T_{j}^{r}, x\right)$, contains at least two sets. The statement now follows from the fact that exactly $n$ players participate in the game.

THEOREM 4.2. In order that a payoff vector $x$ belong to the kernel of a game $G$, it is necessary and sufficient that the players who are separated out at each stage of the profile generated by $x$ receive a zero payment in $x$.

Proof. Let $x \in \mathscr{K}$. Suppose that a player $l$ belongs to an $F_{j}^{r+1}\left(\mathscr{D}\left(T_{j}^{r}, x\right)\right)$ of the profile. There exists a player $k$ in $T_{j}^{r}$ who separated $l$ out by $\mathscr{D}\left(T_{j}^{r}, x\right)$. Note that $k$ and $l$ belong to the same equivalence classes of the 1st, $2 n d, \cdots, r$ th stages of the profile; hence none of the coalitions of $\mathscr{T}_{k, l}$ and $\mathscr{T}_{l, k}$ has an excess greater than $s_{r}(x)$. (See (4.7).) Moreover, since $k$ has separated $l$ out by $\mathscr{D}\left(T_{j}^{r}, x\right)$, it follows from (4.4) and (4.5) that $s_{k, l}=s_{r}(x)$ and $s_{l, k}<s_{r}(x)$. Thus $x_{l}=0$, because $x \in \mathscr{K}$.

We omit the proof of the converse statement, since it will be a direct consequence of Theorem 4.3 (see Remark 4.4).

We shall now generate "abstract" profiles which depend only on the set $N$ of the players and not on the particular game and the particular characteristic function.

Starting from the set $q_{1}=\{N\}$, we shall define inductively sets $q_{2}, q_{3}, \cdots, q_{n}$ and vectors $\delta_{1}, \delta_{2}, \cdots, \delta_{n-1}$ as follows: Suppose that the set $q_{r}=\left\{T_{1}^{r}, T_{2}^{r}, \cdots, T_{u_{r}}^{r}\right\}$ has been defined, where $T_{j}^{r}, j=1,2, \cdots, u_{r}$, are disjoint subsets of $N$. From each class $\mathscr{C}\left(T_{j}^{r}\right), j=1,2, \cdots, u_{r}$, choose a subset $\mathscr{D}\left(T_{j}^{r}\right)$, such that, if $\mathscr{C}\left(T_{1}^{r}\right) \cup \mathscr{C}\left(T_{2}^{r}\right) \cup \cdots \cup \mathscr{C}\left(T_{u_{r}}^{r}\right)$ is not empty, at least one of the sets $\mathscr{D}\left(T_{j}^{r}\right), j=1,2, \cdots, u_{r}$, is not empty. Introduce the notation:

$$
\begin{gathered}
\mathscr{D}\left(q_{r}\right)=\mathscr{D}\left(T_{1}^{r}\right) \cup \mathscr{D}\left(T_{2}^{r}\right) \cup \cdots \cup \mathscr{D}\left(T_{u_{r}}^{r}\right), \\
\delta_{r}=\delta_{r}\left(q_{r}\right)=\left(\mathscr{D}\left(T_{1}^{r}\right), \mathscr{D}\left(T_{2}^{r}\right), \cdots, \mathscr{D}\left(T_{u_{r}}^{r}\right)\right) .
\end{gathered}
$$

Each $\mathscr{D}\left(T_{j}^{r}\right), j=1,2, \cdots, u_{r}$, induces a partition

$$
\left\{T_{j, 1}^{r+1}, T_{j, 2}^{r+1}, \cdots, T_{j, \nu_{j}}^{r+1}, F_{j}^{r+1}\left(\mathscr{D}\left(T_{j}^{r}\right), T_{j}^{r}\right)\right\}
$$

on the set $T_{j}^{r}$ (see (3.5).) Renumber all the equivalence classes in these partitions lexicographically in the lower indices and thus obtain the set $q_{r+1}=\left\{T_{1}^{r+1}, T_{2}^{r+1}, \cdots, T_{u_{r+1}}^{r+1}\right\}$. Continue with this process until you arrive at $q_{n}$. The set

$$
P=P\left(\delta_{1}, \delta_{2}, \cdots, \delta_{n-1}\right)=\left\{q_{1} ; q_{2}, F_{1}^{2} ; \cdots ; q_{n}, F_{1}^{n}, \cdots, F_{u_{n-1}}^{n}\right\}
$$

is called the profile generated by the sequence $\delta_{1}, \delta_{2}, \cdots, \delta_{n-1}$. 
LEMMA 4.1a. Let Pbe a profile generated by a sequence $\delta_{1}, \delta_{2}, \cdots, \delta_{n-1}$. The members of $q_{n}$ are single players.

The proof is similar to the proof given in Lemma 4.1.

Let $P$ be a profile over a set of players $N$, generated by a sequence $\delta_{1}, \delta_{2}, \cdots, \delta_{n-1}$. Consider a game $(N ; v)$. For each set

$$
\mathscr{D}\left(T_{j}^{r}\right), r=1,2, \cdots, n-1, j=1,2, \cdots, u_{r},
$$

consider the set $X_{1}\left(\mathscr{D}\left(T_{j}^{r}\right), T_{j}^{r}\right)$ of payoff vectors that have vanishing coordinates for the players in $F\left(\mathscr{D}\left(T_{j}^{r}\right), T_{j}^{r}\right)$, and, moreover, attain in the coalitions of $\mathscr{D}\left(T_{j}^{r}\right)$ an excess which is maximal for the coalitions in $\mathscr{C}\left(T_{j}^{r}\right)$ i.e.,

$$
\begin{array}{r}
X_{1}\left(\mathscr{D}\left(T_{j}^{r}\right), T_{j}^{r}\right)=\{x: x \in X(N), e(S, x) \geqq e(T, x) \\
\text { whenever } S \in \mathscr{D}\left(T_{j}^{r}\right) \text { and } T \in \mathscr{C}\left(T_{j}^{r}\right), \\
\left.x_{i}=0 \text { for } i \in F\left(\mathscr{D}\left(T_{j}^{r}\right), T_{j}^{r}\right)\right\} .
\end{array}
$$

Clearly, $X_{1}\left(\mathscr{D}\left(T_{j}^{r}\right), T_{j}^{r}\right)=X(N)$ (see $\left.(2.4)\right)$ if $\mathscr{D}\left(T_{j}^{r}\right)$ is empty.

THEOREM 4.3. If $\delta_{1}, \delta_{2}, \cdots, \delta_{n-1}$ is a sequence generating a profile $P$ over a set of players $N$ who participate in a game $(N ; v)$ then

$$
\bigcap_{r=1}^{n-1} \bigcap_{j=1}^{u_{r}} X_{1}\left(\mathscr{D}\left(T_{j}^{r}\right), T_{j}^{r}\right) \subset \mathscr{K}
$$

Proof. Let $x$ belong to the left hand side of (4.11). Suppose that there exist players $k, l$ such that $k \nsim l$ with respect to $x$. We shall prove by induction on $r$ that in each $q_{r}=\left\{T_{1}^{r}, T_{2}^{r}, \cdots, T_{u_{r}}^{r}\right\}$ in $P, r=1,2, \cdots, n$, there exists a set $T_{j}^{r}$ which contains both players, contrary to Lemma 4.1a. This is certainly true for $r=1$ since $q_{1}=$ $\{N\}$. Suppose it is true for a particular $r$, and let $k$ and $l$ belong to $T_{j}^{r}$. Both $s_{k, l}$ and $s_{l, k}$ are attained in coalitions of the class $\mathscr{C}\left(T_{j}^{r}\right)$, because $\mathscr{T}_{k, l} \subset \mathscr{C}\left(T_{j}^{r}\right)$ and $\mathscr{T}_{l, k} \subset \mathscr{C}\left(T_{j}^{r}\right)$. Consider the set $\mathscr{D}\left(T_{j}^{r}\right)$. If $k$ and $l$ are separated by $\mathscr{D}\left(T_{j}^{r}\right)$, then by (4.10), $s_{k, l}=s_{l, k}$, because $x \in X_{1}\left(\mathscr{D}\left(T_{j}^{r}\right), T_{j}^{r}\right)$. If one of the players, say player $l$, is separated out by $\mathscr{D}\left(T_{j}^{r}\right)$ and the other is not separated out, then, by $(4.10)$,

$$
s_{k, l}=\operatorname{Max}_{S \in \mathscr{C}\left(T_{j}^{r}\right)} e(S, x),
$$

and therefore $s_{k, l} \geqq s_{l, k}$. Moreover, by (4.10), $x_{l}=0$. If both players are separated out by $\mathscr{D}\left(T_{j}^{r}\right)$, then $x_{k}=x_{l}=0$. In all these cases $k \approx l$ with respect to $x$, contrary to our assumption. Thus, both $k$ and $l$ are inseparable by $\mathscr{D}\left(T_{j}^{r}\right)$, and therefore belong to the same set in $q_{r+1}$. This concludes the proof. 
REMARK 4.4. Note that it may well happen that $x$ belongs to the right hand side of (4.11) and yet $P$ is not a profile generated by $x$. We allow in (4.10) that $x$ attains maximum excess (for the coalitions in $\left.\mathscr{C}\left(T_{j}^{r}\right)\right)$ also in coalitions outside of $\mathscr{D}\left(T_{j}^{r}\right)$. Moreover, this maximum excess need not be equal to $s_{r}(x)$ (see (4.6)); i.e., it need not be the same for all the classes $\mathscr{C}\left(T_{j}^{r}\right), j=1,2, \cdots, u_{r}$. However, if $P(x)$ is a profile generated by a payoff vector $x$ and if $x$ satisfies the conditions of Theorem 4.2 then $x$ belongs to

$$
\bigcap_{r=1}^{n-1} \bigcap_{j=1}^{u_{r}} X_{1}\left(\mathscr{D}\left(T_{j}^{r}, x\right), T_{j}^{r}\right)
$$

where $\mathscr{D}\left(T_{j}^{r}, x\right)$ are defined by (4.5). Consequently, $x$ belongs to $\mathscr{K}$. This completes the proof of Theorem 4.2.

It will be most useful to combine both theorems in the following fashion: Starting with $q_{1}=\{N\}$, let $\vartheta\left(q_{1}\right)$ be the set of all the nonempty subsets $\mathscr{D}(N)$ of $\mathscr{C}(N)$. Each $\delta_{1}=(\mathscr{D}(N))$ defines a $q_{2}=q_{2}\left(\delta_{1}\right)=\left\{T_{1}^{2}, T_{2}^{2}, \cdots, T_{u_{2}}^{2}\right\}$. Suppose that we have arrived at a $q_{r}=q_{r}\left(\delta_{r-1}\right)=\left\{T_{1}^{r}, T_{2}^{r}, \cdots, T_{u_{r}}^{r}\right\}$, let $\vartheta\left(q_{r}\right)$ be the set of all possible vectors $\delta_{r}$ (see (4.8) and (4.9)). Each element of $\vartheta\left(q_{r}\right)$ generates a $q_{r+1}=q_{r+1}\left(\delta_{r}\right)$, and so on. We obtain a sequence $\vartheta\left(q_{1}\right), \vartheta\left(q_{2}\right), \cdots, \vartheta\left(q_{n-1}\right)$. For each element $\delta_{r}$ in a $\vartheta\left(q_{r}\right)$ we introduce the notation

$$
X_{1}\left(\delta_{r}, q_{r}\right)=\bigcap_{j=1}^{u_{r}} X_{1}\left(\mathscr{D}\left(T_{j}^{r}\right), T_{j}^{r}\right) .
$$

THEOREM 4.5. The kernel of a game $G$ is given by

$$
\mathscr{K}(G)=\bigcup_{\delta_{1} \in \vartheta\left(q_{1}\right)} \bigcup_{\delta_{2} \in \vartheta\left(q_{2}\right) \cdots \delta_{n-1} \in \vartheta\left(q_{n-1}\right)} \bigcap_{r=1}^{n-1} X_{1}\left(\delta_{r}, q_{r}\right) .
$$

The above results hold with a few obvious modifications if the coalition structure is different from $N$. For example, Theorem 4.5 generalizes as follows:

THEOREM 4.6. Let $G$ be an n-person cooperative game and let $\mathscr{B}$ be a coalition structure in G. An i.r.p.c. (x; $\mathscr{B})$ (see (2.3)) belongs to $\mathscr{L}(G)$ if and only if $x$ belongs to the right hand side of (4.14), where the inductive definition of the various symbols starts with $q_{1}=\mathscr{B}$ and $X(N)$ in (4.10) is replaced by $X(\mathscr{B})$ (see (2.4)).

5. Existence of the kernel. Intersection with the core. Using the Brouwer fixed point theorem, it has been proved in [4] that for each coalition structure $\mathscr{B}$ there exists a payoff vector $x$ such that $(x, \mathscr{B}) \in \mathscr{K}$. (See also [9].) In the first part of this section we shall prove this result using only algebraic methods. Again, only 
the case of the coalition structure $N$ will be treated, but a similar proof holds in the general case.

Lemma 5.1. Let $(N ; v)$ be an n-person game and let $\mathscr{C}$ be a fixed set of coalitions. Let $\pi$ be a closed convex polyhedron in $X(N)$ (see (2.4)), then there exists a point $\xi$ such that

$$
\underset{x \in \pi}{\operatorname{Min}} \operatorname{Max}_{S \in \mathscr{C}^{*}} e(S, x)=\underset{S \in \mathscr{C}}{\operatorname{Max}} e(S, \xi) \text {. }
$$

Proof. For each point $x$ in $\pi$ there exists a coalition in $\mathscr{C}$ for which $e(S, x)$ is maximal, because $\mathscr{C}$ is a finite set. Consequently, if $\mathscr{C}=\left\{S_{1}, S_{2}, \cdots, S_{m}\right\}$ there exists a covering $\left\{\pi_{1}, \pi_{2}, \cdots, \pi_{m}\right]$ of $\pi$ such that $\pi_{j}$ consists of points $x$ in $\pi$ for which $\operatorname{Max}_{S} e(S, x)$ is attained in $S_{j}, j=1,2, \cdots, m$. Each $\pi_{j}$ is a closed convex polyhedron since it is given by a system of linear weak inequalities. ${ }^{5}$ Clearly, $\operatorname{Min}_{x \in \pi_{j}} e\left(S_{j}, x\right)$ is attained at a vertex of $\pi_{j}$; hence the required point $\xi$ is one of a finite set of points.

THEOREm 5.2. Let $(N ; v)$ be a cooperative game. There exists a point $x$ such that $x \in \mathscr{K}$.

Proof. Let $\xi^{1}$ be a point in $X(N)$ such that

$$
\operatorname{Min}_{x \in X(N)} \operatorname{Max}_{S \in \mathscr{Q}(N)} e(S, x)=\underset{S \in \mathscr{Q}(N)}{\operatorname{Max}} e\left(S, \xi^{1}\right),
$$

and such that the number of coalitions in $\mathscr{C}(N)$ which attain this maximum excess with respect to $\xi^{1}$ is the smallest possible.

Let $\left\{T_{1}^{2}, T_{2}^{2}, \cdots, T_{u_{2}}^{2} F_{1}^{2}\left(\mathscr{D}\left(N, \xi^{1}\right), N\right)\right\}$ be the second stage of the profile generated by $\xi^{1}$, (the first stage being $\left.\left\{T_{1}^{1}\right\}=\{N\}\right)$. (See (4.2).) We shall show that $\xi_{l}^{1}=0$ for $i \in F_{1}^{2}\left(\mathscr{D}\left(N, \xi^{1}\right), N\right)$. Suppose $\xi_{l}^{1}>0$ for $l$ in $F_{1}^{2}\left(\mathscr{D}\left(N, \xi^{1}\right), N\right)$; we look for a player $k$ in a $T_{\nu}^{2}$, who separates player $l$ out by $\mathscr{D}\left(N, \xi^{1}\right)$. Note that $s_{k, l}\left(\xi^{1}\right)=s_{1}\left(\xi^{1}\right)>s_{l, k}\left(\xi^{1}\right)$ (see (4.3)). Player $k$ demands and receives from player $l$ the positive amount ${ }^{6}$ $\operatorname{Min}\left(\left(s_{k, l}\left(\xi^{1}\right)-s_{l, k}\left(\xi^{1}\right)\right) / 2, \xi_{l}^{1}\right)$. Examining the change which results on the various excesses, we find that:

(i) the excess does not change for the coalitions which either contain both $k$ and $l$ or contain neither of these players.

(ii) it strictly decreases for the coalitions in $\mathscr{T}_{k, l}$.

(iii) it strictly increases for the coalitions in $\mathscr{T}_{l, k}$, but it still remains less than $s_{1}\left(\xi^{1}\right)$ for them.

Denote by $\xi^{1^{*}}$ the new payoff vector, we observe that $\xi^{1^{*}} \in X(N)$,

${ }^{4}$ We are indebted to $\mathrm{M}$. Rabin for the very simple algebraic proof given here.

${ }^{5}$ See D. Gale [5].

${ }^{6}$ Any positive amount smaller than this one will also do. 
and that the number of coalitions which have received the excess $s_{1}\left(\xi^{1}\right)$ strictly decreased. Thus, either $s_{1}\left(\xi^{1^{*}}\right)<s_{1}\left(\xi^{1}\right)$, or $s_{1}\left(\xi^{1^{*}}\right)=s_{1}\left(\xi^{1}\right)$ but fewer coalitions attain this excess with respect to $\xi^{1^{*}}$ than with respect to $\xi^{1}$. Both cases are impossible because of the choice of $\xi^{1}$. The contradiction shows that $\xi_{l}^{1}=0$.

Define $q_{1}=\{N\}, \delta_{1}=\left(\mathscr{D}\left(N, \xi^{1}\right)\right)$ and construct $X_{1}\left(\delta_{1}, q_{1}\right)$. Clearly, $X_{1}\left(\delta_{1}, q_{1}\right)$ is not empty, because it contains the point $\xi^{1}$.

We proceed by induction: Suppose that $\delta_{1}, \delta_{2}, \cdots, \delta_{r-1}, 1 \leqq r \leqq n-1$, have been defined, such that they determine the first $r$ stages of a profile and such that $X_{1}^{r-1}=\bigcap_{i=1}^{r-1} X_{1}\left(\delta_{i}, q_{i}\right) \neq \phi$. Let $\xi^{r}$ be a point in $X_{1}^{r-1}$ such that

$$
\operatorname{Min}_{x \in X_{1}^{r-1}} \operatorname{Max}_{S \in \mathscr{S}^{\left(q_{r}\right)}} e(S, x)=\operatorname{Max}_{S \in \mathscr{C}^{\left(q_{r}\right)}} e\left(S, \xi^{r}\right),
$$

and such that the number of coalitions in $\mathscr{C}\left(q_{r}\right)$ which attain this maximum excess, $s_{r}\left(\xi^{r}\right)$, with respect to $\xi^{r}$ is the smallest possible. These coalitions form the class $\mathscr{D}\left(q_{r}, \xi^{r}\right)$ which determines the $u_{r}$-tuple $\delta_{r}\left(\xi^{r}\right)=\left(\mathscr{D}\left(T_{1}^{r}, \xi^{r}\right), \cdots, \mathscr{D}\left(T_{u_{r}}^{r}, \xi^{r}\right)\right)($ see $(4.5))$. Each set $\mathscr{D}\left(T_{j}^{r}, \xi^{r}\right), j=$ $1,2, \cdots, u_{r}$, induces a partition $\left.T_{j, 1}^{r+1}, T_{j, 2}^{r+1}, \cdots, T_{j, \nu_{j}}^{r+1} ; F_{j}^{r+1} \mathscr{D}\left(T_{j}^{r}, \xi^{r}\right), T_{j}^{r}\right)$ of $T_{j}^{r}$.

As in the first stage, we shall show, by arriving at a contradiction, that $\xi_{i}^{r}=0$ for $i \in F_{j}^{r+1}\left(\mathscr{D}\left(T_{j}^{r}, \xi^{r}\right), T_{j}^{r}\right)$. Indeed, if there exists a player $l$ who has been separated out by $\mathscr{D}\left(T_{j}^{r}, \xi^{r}\right)$, and $\xi_{l}^{r}>0$, we look for player $k$ in a $T_{j, \nu}^{r+1}$ who has separated player $l$ out. The players $k$ and $l$ were inseparable in the first $r$ stages of the profile. In particular, $k, l \in T_{j}^{r}$. Since $\mathscr{T}_{k, l} \subset \mathscr{C}\left(T_{j}^{r}\right) \subset \mathscr{C}\left(q_{r}\right)$ and $\mathscr{T}_{l, k} \subset \mathscr{C}\left(T_{j}^{r}\right) \subset \mathscr{C}\left(q_{r}\right)$, it follows that $s_{k, l}\left(\xi^{r}\right)=s_{r}\left(\xi^{r}\right)$ and $s_{l, k}\left(\xi^{r}\right)<s_{r}\left(\xi^{r}\right)$. Thus, player $k$ can demand and receive from player $l$ the positive amount

$$
\operatorname{Min}\left(\left(s_{k, l}\left(\xi^{r}\right)-s_{l, k}\left(\xi^{r}\right)\right) / 2, \xi_{l}^{r}\right) \text {. }
$$

We shall first show that the resulting payof vector $\xi^{* *}$ is in $X_{1}^{r-1}$. Indeed, since $\mathscr{C}\left(q_{1}\right) \supset \mathscr{C}\left(q_{2}\right) \supset \cdots \supset \mathscr{C}\left(q_{r}\right)$, it follows that $s_{1}\left(\xi^{r}\right) \geqq$ $s_{2}\left(\xi^{r}\right) \geqq \cdots \geqq s_{r}\left(\xi^{r}\right)$. The transition from $\xi^{r}$ to $\xi^{r^{*}}$ does not affect the excesses of the coalitions in $\mathscr{C}\left(q_{\nu}\right), \nu=1,2, \cdots, r-1$, since these coalitions either contain $k$ and $l$ or contain neither of them. On the other hand, no coalition's excess in $\mathscr{C}\left(q_{r}\right)$ increases beyond $s_{r}\left(\xi^{r}\right)$. Consequently, the excesses of the various coalitions in $\mathscr{D}\left(q_{\nu}\right), \nu=1,2, \cdots, r-1$, remain maximal with respect to $\xi^{* *}$. Moreover, players who were separated out in the first $r$ stages continue to receive a zero payment in $\xi^{r *}$.

Just as in the case of $\xi^{1}$ we find that either $s_{r}\left(\xi^{r^{*}}\right)<s_{r}\left(\xi^{r}\right)$ or $s_{r}\left(\xi^{*}\right)=s_{r}\left(\xi^{r}\right)$ but fewer coalitions attain it with respect to $\xi_{r}^{*}$; hence the desired contradiction.

Defining $\delta_{r}=\delta_{r}\left(\xi^{r}\right)$, we have proved that $X_{1}^{r}=\bigcap_{i=1}^{r} X_{1}\left(\delta_{i}, q_{i}\right)$ is 
not empty, since it contains the point $\xi^{r}$. Continue until $r=n-1$, and you obtain, by Theorem 4.3, a nonempty polyhedron in the kernel of the game.

Unlike the proof given in [4], this proof shows directly that if the coefficients of the characteristic function are taken from any ordered field, then there exists a point in the kernel whose coordinates are in the ordered field ${ }^{7}$. The same method of proof can be used to obtain other properties of the kernel, as we shall presently see. We shall start with the concept of the $\varepsilon$-core which has been introduced by L. S. Shapley and M. Shubik ${ }^{8}$ [12].

Definition 5.3. The $\varepsilon$-core, $\varepsilon \geqq 0$, in a game $(N ; v)$ is the set of payoff vectors $x$ which satisfy

$$
e(S, x) \leqq \varepsilon \quad \text { for each coalition } S .
$$

The 0-core is, of course, the usual core of the game.

THEOREM 5.4. If the e-core of a game is not empty, it contains a point in the kernel.

Proof. Let $C$ be the $\varepsilon$-core. The proof runs exactly as the proof of Theorem 5.2, except that $X(N)$ in (5.2) should be replaced by $X(N) \cap C$, and $X_{1}^{r-1}$ in (5.3) should be replaced by $X_{1}^{r-1} \cap C$. The only thing that should be clarified is that the transition from $\xi^{r}$ to $\xi^{r^{*}}$ does not take us out of the $\varepsilon$-core. This is indeed the case, since $s_{1}\left(\xi^{*}\right) \leqq s_{1}\left(\xi^{r}\right) \leqq \varepsilon$.

Theorem 5.4 is quite surprising since there is no apparent a priori connection between the core conception and the ideas involved in the kernel. One can easily construct games with nonempty core in which the kernel contains points outside the core. Other games exist, where the kernel is properly contained in the core. Observing that the bargaining set $\mathscr{C}_{1}^{(i)}$ contains the core $^{10}$ and that the kernel is a subset of $\mathscr{L}_{1}^{(i)}$, suggests that the "reason" that Theorem 5.4 works should be

${ }^{7}$ An indirect proof runs as follows: The inequalities (2.8) which determine the kernel can be written as a system of linear inequalities in the variables $v(B)$ and $x_{i}$, with coefficients which are $-1,0$ or 1 . The inequalities are connected by the words "and" and "or". By projection, we can successively eliminate the variables (see e.g., [1] ). The existence of a point in the kernel is therefore equivalent to the assertion that at least one of a set of inequalities $\alpha_{1} \geq \beta_{1}, \cdots, \alpha_{m} \geq \beta_{m}$ involving rational numbers is true. The fact that it is true is a consequence of any existence proof whether algebraic or not, and for any particular ordered field. This implies the existence in every ordered field.

8 Shapley and Shubik use the term "strong $\varepsilon$-core".

${ }^{9}$ If one defines an $\varepsilon$-core for $\varepsilon<0$, by restricting (5.4) to the coalitions of $\varepsilon(N)$, the theorem is still true.

${ }_{10}$ An obvious modification of the bargaining set contains the $\varepsilon$-core. 
found by studying the location of the kernel in the bargaining set. Such a study, we believe, is worthwhile.

6. The maximal dimension of the kernel. Let $G$ be an $n$-person game. The kernel $\mathscr{K}(G)$ is a finite union of closed and convex polyhedra:

$$
\mathscr{K}(G)=C_{1} \cup C_{2} \cup \cdots \cup C_{\mu} .
$$

The dimension $d(\mathscr{K}(G))$ of $\mathscr{K}(G)$ is defined to be the dimension of a highest dimensional polyhedron $C_{i}$; i.e.,

$$
d(\mathscr{K}(G))=\operatorname{Max} d\left(C_{i}\right), i=1,2, \cdots, \mu .
$$

The dimension of $\mathscr{K}(G)$ does not depend, of course, on the particular choice of polyhedra whose union is the kernel.

There arises the problem of determining how high the dimension can be for a given $n$. Accordingly, we define:

$$
d(n)=\operatorname{Max}\{d(\mathscr{K}(G)): G \text { runs over all } n \text {-person games }\} .
$$

In this section we shall prove that ${ }^{11} d(n)=n-2-\left[\log _{2}\left(n-\frac{1}{2}\right)\right]$, and at the end of the section we shall give sharp bounds to the dimension of the kernel for coalition structures different from $N$.

The proof itself applies the results of Section 4, yet it is carried on through a series of lemmas, interesting for their own sake. The main result is of theoretical as well as of computational importance.

Let $N=\{1,2, \cdots, n\}$ and let $S$ be a subset of $N$ (which may be the empty set). We denote by $\chi^{S}$ the characteristic vector of $S$; i.e., $\chi^{S}=\left(\chi_{1}^{S}, \chi_{2}^{S}, \cdots, \chi_{n}^{S}\right)$, where $\chi_{i}^{S}=1$ if $i \in S$ and $\chi_{i}^{S}=0$ if $i \notin S$.

If $Q^{\circ}$ is a set of subsets of $N$, we denote generically by $Q^{*}$ the set of the characteristic vectors of the elements of $\mathbb{Q}^{\infty}$; i.e., $\mathbb{Q}^{*}=\left\{\chi^{S}: S \in \mathbb{Q}^{\mathbb{2}}\right\}$.

Definition 6.1. A nonempty set $\mathscr{D}$ of subsets of $N$ will be called completely separating, if every two players of $N$ separate each other by $\mathscr{D}$. It is called minimal completely separating if no proper subset of $\mathscr{D}$ is completely separating.

The following lemma is of basic importance.

LeMma 6.2. Let $\mathscr{D}$ be a nonempty completely separating set of coalitions of $N=\{1,2, \cdots, n\}$. There exist $\lambda_{n}=2+\left[\log _{2}\left(n-\frac{1}{2}\right)\right]$ linearly independent vectors $\alpha^{(1)}, \alpha^{(2)}, \cdots, \alpha^{\left(\lambda_{n}\right)}$ in $\mathscr{D}^{*}$, such that if $\chi^{N}=c_{1} \alpha^{(1)}+\cdots+c_{n} \alpha^{\left(\lambda_{n}\right)}$ then $c_{i} \geqq 0, i=1,2, \cdots, \lambda_{n}$.

${ }^{11}[a]$ will denote in this section the integral part of $a$. 
Proof. For $n=1$ the lemma is obvious. If $n=2$, then $\mathscr{D}^{*}$ must contain the $\lambda_{2}=2$ vectors $(1,0)$ and $(0,1)$. These are linearly independent and $(1,1)=(1,0)+(0,1)$. We continue by induction. Suppose the lemma is true for all the values $n_{1}, n_{1}\langle n, n\rangle 2$ and let $\mathscr{D}$ satisfy the conditions of the lemma. Without loss of generality, we may assume that $\mathscr{D}$ is a minimal completely separating set. Let $\alpha=\chi^{T} \in \mathscr{D}^{*}$. We distinguish two cases:

Case 1. $|T| \geqq[(n+1) / 2]$, where $|T|$ denotes the number of players in $T$. We restrict ourselves to the players of $T$ and form the set $\mathscr{D}_{T}=(\mathscr{D} / T)-\{T\}=\{S \cap T: S \in \mathscr{D}, S \cap T \neq T\} . \quad \mathscr{D}_{T}$ is a completely separating set of subsets of $T$ (not necessarily minimal). By the induction hypothesis there exist $\lambda_{|T|}|T|$-dimensional vectors, $\bar{\alpha}^{(1)}, \bar{\alpha}^{(2)}, \cdots, \bar{\alpha}^{(\lambda|T|)}$, in $\mathscr{D}_{T}{ }^{*}$ which are linearly independent and such that if $\chi^{T}=c_{1} \bar{\alpha}^{(1)}+\cdots+c_{\lambda_{|T|}} \bar{\alpha}^{(\lambda|T|)}$ then $c_{i} \geqq 0, i=1,2, \cdots, \lambda_{|T|}$. Each vector $\bar{\alpha}^{(i)}$ is a restriction of at least one vector $\alpha^{(i)}$ in $\mathscr{D}^{*}$ to the players of $T$; i.e., $\bar{\alpha}^{(i)}=\alpha^{(i)} / T$ where $\alpha^{(i)} \in \mathscr{D}^{*}, i=1,2, \cdots, \lambda_{|T|}$. Having fixed one such choice, let $\alpha^{(1)}=\chi^{T_{1}}, \cdots, \alpha^{(u)}=\chi^{T_{u}}$, where $u=$ $\lambda_{|T|}$, we shall prove that $\alpha, \alpha^{(1)}, \cdots, \alpha^{\left(\lambda\left|T^{T}\right|\right)}$ are linearly independent. Indeed, $\alpha^{(1)}, \cdots, \alpha^{(\lambda|T|)}$ are linearly independent. If

$$
\alpha=c_{1} \alpha^{(1)}+\cdots+c_{\lambda_{|T|}} \alpha^{\left(\lambda_{|T|}\right)},
$$

then, since $\chi^{T}=\sum_{i=1}^{\lambda|T|} c_{i} \bar{\alpha}^{(i)}$, it follows that $c_{i} \geqq 0, i=1,2, \cdots, \lambda_{|T|}$. In other words, $\alpha=c_{i_{1}} \alpha^{\left(i_{1}\right)}+\cdots+c_{i_{p}} \alpha^{\left(i_{p}\right)}$ where $p \leqq \lambda_{|T|}$ and $c_{i_{j}}>0$ for $j=1,2, \cdots, p$. Consequently, $\bigcup_{j=1}^{p} T_{i_{j}}=T$, because $\alpha$ has vanishing coordinates for members which are not in $T$. Thus, if $k \in T$ and $l \notin T$, there exists a coalition $T_{i_{j}}, 1 \leqq j \leqq p$, which contains $k$ and does not contain $l$. Consider the set $\mathscr{D}-\{T\}$. Since $\mathscr{D}$ is a completely separating set, it follows that $\mathscr{D}-\{T\}$ is a completely separating set. This contradicts the assumption that $\mathscr{D}$ is a minimal completely separating set.

We shall now prove that if

$$
\chi^{N}=c \alpha+c_{1} \alpha^{(1)}+\cdots+c_{\lambda_{|T|}} \alpha^{\left(\lambda \mid T^{\mid}\right)},
$$

then $c \geqq 0$ and $c_{j} \geqq 0, j=1, \cdots, \lambda_{|T|}$.

If (6.4) holds, then $\chi^{T}=c \chi^{T}+c_{1} \bar{\alpha}^{(1)}+\cdots+c_{\lambda_{\mid T_{\mid}}} \bar{\alpha}^{\left(\lambda_{|T|}\right)}$, hence $(1-c) \chi^{T}=c_{1} \bar{\alpha}^{(1)}+\cdots+c_{\lambda_{|T|}} \bar{\alpha}^{\left(\lambda_{|T|}\right)}$.

Consequently, by the induction hypotheses, either $c \leqq 1$ and $c_{i} \geqq 0$ or $c>1$ and $c_{i} \leqq 0$, for all $i, i=1,2, \cdots, \lambda_{\left|T^{\mid}\right|}$. By the minimal property of $\mathscr{D}, \alpha$ must have at least one vanishing coordinate; therefore, it is not possible that some of the $c_{i}$ 's are negative and the rest take a zero value. Consequently, $c_{i} \geqq 0$ for all $i, i=1,2, \cdots, \lambda_{\mid T !}$. There remains to show that $c \geqq 0$. Let $k \in T$ and $l \notin T$. If $c<0$, then, by 
(6.4), $\sum_{i=1}^{\lambda_{i=1} \mid} c_{i} \alpha_{k}^{(i)}>1=\sum_{i=1}^{\lambda_{1}|T|} c_{i} \alpha_{l}^{(i)}$, where $\alpha_{p}^{(i)}$ is the $p$-th coordinate of $\alpha^{(i)}$. Since all the $c_{i}^{\prime}$ 's are nonnegative, it follows that there exists a coalition $T_{j}, 1 \leqq j \leqq \lambda_{|T|}$, which contains player $k$ and does not contain player $l$. As previously, this implies that $\mathscr{D}-\{T\}$ is a completely separating set, contrary to the minimal property of $\mathscr{D}$.

Finally, since $|T| \geqq[(n+1) / 2]$, it follows that $\lambda_{\left|T^{\prime}\right|} \geqq \lambda_{n}-1$. This proves the lemma for case 1 .

Case 2. $|N-T| \geqq[(n+1) / 2]$. We restrict ourselves to the players $N-T$ and form the set $\mathscr{D}_{N \rightarrow T}=\{S \cap(N-T): S \in \mathscr{D}\} . \quad$ By the induction hypothesis there exist $\lambda_{p}$ linearly independent vectors $\bar{\alpha}^{(1)}, \cdots, \bar{\alpha}^{\left(\lambda_{p}\right)}$ in $\mathscr{D}^{*}{ }_{N-T}$, where $p=|N-T|$, and such that if $\chi^{N-T}=$ $c_{1} \bar{\alpha}^{(1)}+\cdots+c_{\lambda_{p}} \bar{\alpha}^{\lambda_{p}}$ then $c_{i} \geqq 0, i=1,2, \cdots, \lambda_{p}$. We choose vectors $\alpha^{(1)}=$ $\chi^{T_{1}}, \cdots, \alpha^{(u)}=\chi^{T_{u}}$ in $\mathscr{D}^{*}$, where $u=\lambda_{p}$, such that $\alpha^{(i)} / N-T=\bar{\alpha}^{(i)}$, $i=1,2, \cdots, \lambda_{p}$. Clearly, $\alpha, \alpha^{(1)}, \cdots, \alpha^{\left(\lambda_{p}\right)}$ are linearly independent, for, otherwise, $\alpha=c_{1} \alpha^{(1)}+\cdots+c_{\lambda_{p}} \alpha^{\left(\lambda_{p}\right)}$, and, since $\alpha$ has vanishing coordinates for indices in $N-T, 0=c_{1} \bar{\alpha}^{(1)}+\cdots+c_{\lambda_{p}} \bar{\alpha}^{\left(\lambda_{p}\right)}$, which is impossible.

If $\chi^{N}=c \alpha+c_{1} \alpha^{(1)}+\cdots+c_{\lambda_{p}} \alpha^{\left(\lambda_{p}\right)}$ then $\chi^{N-T}=c_{1} \bar{\alpha}^{(1)}+\cdots+c_{\lambda_{p}} \bar{\alpha}^{\left(\lambda_{p}\right)}$; hence, by the induction hypothesis, $c_{i} \geqq 0, i=1,2, \cdots, \lambda_{p}$. If $c<0$, then an argument similar to the one given in case 1 shows that $\mathscr{D}-\{T\}$ is a completely separating set, contrary to the minimal property of $\mathscr{D}$. As in case 1 , since $|N-T| \geqq[(n+1) / 2]$, it follows that $\lambda_{p} \geqq$ $\lambda_{n}-1$. This proves the lemma also in the second case.

COROLLARY 6.3. If $\mathscr{D}$ is a nonempty completely separating set of subsets of $N=\{1,2, \cdots, n\}, n \geqq 2$, then $\mathscr{D}^{*}$ contains $\lambda_{n}$ vectors $\alpha^{(1)}, \cdots, \alpha^{\left(\lambda_{n}\right)}$, such that the vectors $\alpha^{(2)}-\alpha^{(1)}, \cdots, \alpha^{\left(\lambda_{n}\right)}-\alpha^{(1)}$ are linearly independent and $\chi^{N}$ is not a linear combination of $\alpha^{(i)}-\alpha^{(1)}$, $i=2, \cdots, \lambda_{n}$.

Proof. The vectors $\alpha^{(1)}, \alpha^{(2)}, \cdots \alpha^{\left(\lambda_{n}\right)}$ of Lemma 6.2 satisfy these requirements. Indeed, $\alpha^{(2)}-\alpha^{(1)}, \cdots, \alpha^{\left(\lambda_{n}\right)}-\alpha^{(1)}$ are linearly independent. If $\chi^{N}=c_{2}\left(\alpha^{(2)}-\alpha^{(1)}\right)+\cdots+c_{\lambda_{n}}\left(\alpha^{\left(\lambda_{n}\right)}-\alpha^{(1)}\right)$ then

$$
\chi^{N}=\left(-\sum_{i=2}^{n} c_{i}\right) \alpha^{(1)}+c_{2} \alpha^{(2)}+\cdots+c_{\lambda_{n}} \alpha^{\left(\lambda_{n}\right)} .
$$

By Lemma 6.2, the coefficients are nonnegative and, therefore, they all vanish, which is also impossible.

Let $\delta_{1}, \delta_{2}, \cdots, \delta_{n-1}$ be a sequence generating a profile. We know that it generates a polyhedron $\bigcap_{r=1}^{n-1} X_{1}\left(\delta_{r}, q_{r}\right)$ of the kernel. The same polyhedron can be generated by a different sequence $\delta_{1}^{0}, \delta_{2}^{0}, \cdots, \delta_{n-1}^{0}$ having the property that all the components of $\delta_{r}^{0}$, except, perhaps, one, are the empty set, yet, as long as the corresponding 
$q_{r}^{0}=\left\{T_{1}^{0 r}, T_{2}^{0 r}, \cdots, T_{u_{0 r}}^{0 r}\right\}$ does not consist of single players, $\delta_{r}^{0}$ contains a nonempty component. This is done in the following fashion: Renumber the nonempty sets $\mathscr{D}\left(T_{j}^{r}\right), r=1,2, \cdots, n-1, j=1,2, \cdots, u_{r}$ which appear in the $\delta_{r}{ }^{\prime}$ s lexiographically in $r$ and $j$. You obtain a sequence $\mathscr{D}_{1}, \mathscr{D}_{2}, \cdots, \mathscr{D}_{t}$. If $\mathscr{D}_{s}=\mathscr{D}\left(T_{j}^{r}\right)$, set $\delta_{s}^{0}=\left(\varnothing, \cdots, \varnothing, \mathscr{D}_{s}, \varnothing, \cdots, \varnothing\right)$, where $\mathscr{D}_{s}$ is located such that it corresponds to the equivalence class $T_{j}^{r}, s=1,2, \cdots, t,\left(T_{j}^{r}\right.$ must be an element of $\left.q_{s}^{0}\right)$, and the length of the vector $\delta_{s}^{0}$ is equal to the number of elements in $q_{s}^{0}$. Let $\delta_{t+1}^{0}, \delta_{t+2}^{0}, \cdots, \delta_{n-1}^{0}$ be vectors of proper length whose components are the empty sets. Clearly, $\bigcap_{r=1}^{n-1} X_{1}\left(\delta_{r}, q_{r}\right)=\bigcap_{r=1}^{n-1} X_{1}\left(\delta_{r}^{0}, q_{r}^{0}\right)$. Therefore, formula (4.14) remains true if one restricts the various sequences generating profiles to sequences whose elements are vectors which have at most one component different from the empty set. Such sequences will be called elementary sequences.

DEFINITION 6.4. An elementary sequence $\delta_{1}, \delta_{2}, \cdots, \delta_{n-1}$ will be called completely separating, if at no stage is a player separated out.

We shall generalize Corollary 6.3 to completely separating elementary sequences.

Lemma 6.5. Let $\delta_{1}, \delta_{2}, \cdots, \delta_{n-1}$ be a completely separating elementary sequence generating a profile, $n \geqq 2$. Let

$$
\delta_{r}=\left(\varnothing, \cdots, \varnothing, \mathscr{D}_{r}\left(T_{j}^{r}\right), \varnothing, \cdots, \varnothing\right),
$$

where $\mathscr{D}_{r}\left(T_{j}^{r}\right) \neq \varnothing, r=1,2, \cdots, t$, and $\delta_{t+1}, \cdots, \delta_{n-1}$ are vectors whose components are $n$ empty sets. There exists a subsequence of the $\mathscr{D}_{r}\left(T_{j}^{r}\right)^{\prime} \mathrm{s}$, which we renumber $\mathscr{D}_{1}, \mathscr{D}_{2}, \cdots, \mathscr{D}_{\sigma}$, such that there exist vectors $\alpha^{(\rho, i)}, \alpha^{(\rho, i)} \in \mathscr{D}_{\rho}^{*}, \rho=1,2, \cdots, \sigma, i=1,2, \cdots, \nu_{\rho}, \nu_{\rho} \geqq 2$, and

(i) all the vectors $\alpha^{(\rho, i)}-\alpha^{(\rho, 1)}$ are linearly independent, $\rho=$ $1,2, \cdots, \delta, i=2,3, \cdots, \nu_{\rho}$,

(ii) $\chi^{N}$ is not a linear combination of the vectors which appear in (i).

(iii) $\sum_{\rho=1}^{\sigma}\left(\nu_{\rho}-1\right) \geqq \lambda_{n}-1$, where $\lambda_{n}=2+\left[\log _{2}\left(n-\frac{1}{2}\right)\right]$.

Proof. By induction. If $n=2$ then $t=1$. Choose $\mathscr{D}_{1}=\mathscr{D}(N)$, and the lemma follows from Corollary 6.3. Assume that the lemma is true for all $n_{1}, n_{1}<n$, where $n>2$. If $t=1$, the lemma follows again from Corollary 6.3. We therefore assume that $t \geqq 2$. Choose $\mathscr{D}_{1}=\mathscr{D}(N)=\left(T_{1}^{1}\right)$, and select the equivalence class $T_{j_{0}}^{2}$ induced by $\mathscr{D}_{1}$ which consists of the largest number of players, say $p$. If $q$ is the number of the equivalence classes which are induced by $\mathscr{D}_{1}$, then

$$
q \geqq n / p \text { if } p \text { divides } n \text { and } q \geqq[n / p]+1 \text {, otherwise. }
$$


From each equivalence class select a player and restrict the vectors in $\mathscr{D}_{1}^{*}$ to the selected players. By Corollary 6.3 applied to the restricted vectors one deduces that the original $\mathscr{D}_{1}^{*}$ contains $\lambda_{q}$ vectors $\alpha^{(1,1)}, \cdots, \alpha^{\left(1, \nu_{1}\right)}$, where $\nu_{1}=\lambda_{q}$, such that $\alpha^{(1, i)}-\alpha^{(1,1)}$ are linearly independent, $i=2,3, \cdots, \nu_{1}$, and $\chi^{N}$ is not a linear combination of these differences. It follows from (6.5), since $p<n$, that

$$
\nu_{1} \geqq \lambda_{n / p} \text { if } p \text { divides } n ; \nu_{1} \geqq \lambda_{[n / p]+1} \text {, otherwise; hence } \nu_{1} \geqq 2 \text {. }
$$

From the remaining $\delta_{2}, \delta_{3}, \cdots, \delta_{n-1}$ select a subsequence $\delta_{r_{1}}, \delta_{r_{2}}, \cdots, \delta_{r_{p-1}}$ consisting of those $\delta_{r}$ 's whose nonempty components $\mathscr{D}_{r}\left(T_{j}^{r}\right)$ are such that $T_{j}^{r} \subset T_{j_{0}}^{2}$, and, if necessary, $\delta^{\prime}$ s whose components are empty sets. Restrict the consideration to the players in $T_{j_{0}}^{2}$ and form $\delta_{r_{i}} / T_{j_{0}}^{2}=$ $\left(\varnothing, \cdots, \mathscr{D}_{r_{i}}\left(T_{j}^{r_{i}}\right) / T_{j}^{2}, \varnothing, \cdots, \varnothing\right)$, if $\delta_{r_{i}}$ has a nonempty component. Otherwise, $\delta_{r_{i}} / T_{j_{0}}^{2}=(\varnothing, \cdots, \varnothing) ; i=1,2, \cdots, p-1$. Here, $\mathscr{D}_{r_{i}}\left(T_{j}^{r_{i}}\right) / T_{j_{0}}^{2}$ is restriction of $\mathscr{D}\left(T_{j}^{r i}\right)$ to the players in $T_{j_{0}}^{2}$ and the empty components in $\delta_{r_{i}} / T_{j_{0}}^{2}$ correspond only to the partition of $T_{j_{0}}^{2}$. The sequence $\delta_{r_{1}} / T_{j_{0}}^{2}$, $\delta_{r_{2}} / T_{j_{0}}^{2}, \cdots, \delta_{r_{p-1}} / T_{j_{0}}^{2}$ is a completely separating elementary sequence generating a profile for the players $T_{j_{0}}^{2}$; hence, by the induction hypothesis, there exists a subsequence of the $\mathscr{D}_{r}\left(T_{j}^{r}\right)^{\prime} \mathrm{s}, r \geqq 2$, which we renumber as $\mathscr{D}_{2}, \mathscr{D}_{3}, \cdots, \mathscr{D}_{\sigma}$, such that there exists $p$-dimensional vectors $\bar{\alpha}^{(\rho, i)}$, $\bar{\alpha}^{(\rho, i)} \in\left(\mathscr{D}_{\rho} / T_{j_{0}}^{2}\right)^{*}, \rho=2,3, \cdots, \sigma, i=1,2, \cdots, \nu_{\rho}, \nu_{\rho} \geqq 2$, and

(i $)^{\circ}$ all the vectors $\bar{\alpha}^{(\rho, i)}-\bar{\alpha}^{(\rho, 1)}$ are linearly independent, $\rho=2,3, \cdots, \sigma, i=2,3, \cdots, \nu_{\rho}$,

(ii) ${ }^{\circ} \quad \chi^{T^{2}} j_{0}$ is not a linear combination of the vectors which appear in (i) ${ }^{\circ}$,

(iii) $)^{\circ} \quad \sum_{p=2}^{e}\left(\nu_{\rho}-1\right) \geqq \lambda_{p}-1$.

Choose vectors $\alpha^{(\rho, i)}$ in $\mathscr{D}_{\rho}^{*}, \rho=2,3, \cdots, \sigma$, such that $\alpha(\rho, i) / T_{j_{0}}^{2}=\bar{\alpha}^{(\rho, i)}$. We shall prove that the $\left(\alpha^{(\rho, i)}\right)^{\prime} \mathrm{s} \rho=1,2, \cdots, \sigma, i=1,2, \cdots, \nu_{\rho}$, satisfy (i), (ii) and (iii).

If $\sum_{\rho=1}^{\sigma} \sum_{i=2}^{\nu_{\rho}} c_{\rho, i}\left(\alpha^{(\rho, i)}-\alpha^{(\rho, 1)}\right)=0$, then

$$
\sum_{i=2}^{\nu_{1}} c_{1, i}\left(\alpha^{(1,1)} / T_{j_{0}}^{2}-\alpha^{(1, i)} / T_{j_{0}}^{2}\right)=\sum_{\rho=2}^{\sigma} \sum_{i=2}^{\nu_{\rho}} c_{\rho, i}\left(\bar{\alpha}^{(\rho, i)}-\bar{\alpha}^{(\rho, 1)}\right) .
$$

Observe that each of the vectors $\alpha^{(1, i)} / T_{j_{0}}^{2}$ consists of either $p$ zeroes or $p 1^{\prime}$ s, because $T_{j_{0}}^{2}$ is an equivalence class induced by $\mathscr{D}_{1}$. Thus, the left hand side of (6.7) is equal to $c \chi^{r^{2}}{ }_{j_{0}}$, where $c$ is a constant number. By (6.7) and (ii) ${ }^{\circ}, c=0$; hence, by (i) ${ }^{\circ}, c_{\rho, i}=0, \rho=2,3, \cdots, \sigma$, $i=2, \cdots, \nu_{\rho}$. Consequently, also $c_{1, i}=0$ and therefore (i) has been proved.

If $\chi^{N}=\sum_{\rho=1}^{\sigma} \sum_{i=2}^{\nu_{\rho}} d_{\rho, i}\left(\alpha^{(\rho, i)}-\alpha^{(\rho, 1)}\right)$, then

$$
\chi^{T^{2}{ }^{2}}=\sum_{i=2}^{\nu_{1}} d_{1, i}\left(\alpha^{(1, i)} / T_{j_{0}}^{2}-\alpha^{(1,1)} / T_{j_{0}}^{2}\right)+\sum_{\rho=2}^{\sigma} \sum_{i=2}^{\nu_{\rho}} d_{\rho, i}\left(\bar{\alpha}^{(\rho, i)}-\bar{\alpha}^{(\rho, 1)}\right) .
$$

Again, $d_{\rho, i}=0, \rho=2,3, \cdots, \sigma, i=2,3, \cdots, \nu_{\rho}$; hence $\chi^{N}$ is a linear combination of the $\left(\alpha^{(1, i)}-\alpha^{(1,1)}\right)^{\prime}$ s which is impossible. 
In the proof of (iii) we distinguish two cases.

Case 1. $p$ divides $n$. In this case, by (iii) ${ }^{\circ}$ and (6.6), (6.8) $\sum_{\rho=1}^{\sigma}\left(\nu_{\rho}-1\right) \geqq \lambda_{n / p}-1+\lambda_{p}-1=2+\left[\log _{2}\left(n / p-\frac{1}{2}\right)\right]+\left[\log _{2}\left(p-\frac{1}{2}\right)\right]$.

Case 2. $p$ does not divide $n$. In this case, by (iii) ${ }^{\circ}$ and $(6.6)$,

$$
\begin{aligned}
\sum_{\rho=1}^{\sigma}\left(\nu_{\rho}-1\right) & \geqq 2+\left[\log _{2}\left([n / p]+1-\frac{1}{2}\right)\right]+\left[\log _{2}\left(p-\frac{1}{2}\right)\right] \\
& \geqq 2+\left[\log _{2} n / p\right]+\left[\log _{2}\left(p-\frac{1}{2}\right)\right] .
\end{aligned}
$$

A somewhat lengthy but straightforward calculation shows that the right hand sides of (6.8) and (6.9) are greater than or equal to $\lambda_{n}-1$. This completes the proof.

We can now deduce the maximal dimension of the kernel. Actually, we shall first deduce a bound for the dimensions of pseudo kernels of $n$-person games and then exhibit a game with a kernel whose dimension attains this bound. Let $(N ; v)$ be a game where $v(S) \geqq 0$ for each coalition $S$, but now we neither assume that $v(i)=0$ for each $i$ nor do we require that $v(S) \geqq \sum_{i \in S} v(i)$. The pseudo kernel of such a game is literally defined exactly as in $\S 2$. (In particular, the payoff configurations are required to satisfy $x_{i} \geqq 0, i=1,2, \cdots, n$, and not $\left.x_{i} \geqq v(i)\right)$. Pseudo kernels were introduced in [4], \&7. They are indispensable tools for the computation of the kernel (see [2]). Since no use is made in this paper of the fact $v(i)=0$, all the results, and, in particular, the representation formula, hold also for the wider class of pseudo kernels.

TheOREM 6.6. $d(n)=n-2-\left[\log _{2}\left(n-\frac{1}{2}\right)\right]$. (See (6.3).) The same formula holds for the maximal dimension of the pseudo kernel of n-person game.

Proof. Denote, as before, $\lambda_{n}=2+\left[\log _{2}\left(n-\frac{1}{2}\right)\right]$. We shall first show by induction for pseudo kernels that ${ }^{12} d(n) \leqq n-\lambda_{n}$. The theorem is true for $n=1,2,3$, since in these cases the pseudo kernel always consists of a point. Assume that the inequality holds for all $n_{1}<n$, $n>3$. Let $G$ be an $n$-person game whose pseudo kernel attains the maximal dimension. Represent the pseudo kernel $\mathscr{K}(G)$ as a finite union of closed and convex polyhedra of the form $\bigcap_{r=1}^{n-1} X_{1}\left(\delta_{r}, q_{r}\right)$, where $\delta_{1}, \delta_{2}, \cdots, \delta_{n-1}$ is an elementary sequence generating a profile. Let $C=C\left(\delta_{1}, \delta_{2}, \cdots, \delta_{n-1}\right)$ be a fixed polyhedron having a maximal dimension; then $d(n)=d(\mathscr{K}(G))=d(C)$. If $\delta_{1}, \delta_{2}, \cdots, \delta_{n-1}$ is not completely sepa-

${ }^{12}$ I.e., $d(n)$ represents here the maximal dimension of the pseudo kernel. 
rating, then at least one player, say player $l$, is separated out at one stage and therefore $x_{l}=0$ for $x \in C$. By the method of deletion of a player (see [4], §7) we know that $C$ is contained in the pseudo kernel of an $(n-1)$-person game; hence, by the induction hypothesis, $d(n) \leqq$ $(n-1)-\lambda_{n-1}$ and, therefore, $d(n) \leqq n-\lambda_{n}$. If $\delta_{1}, \delta_{2}, \cdots, \delta_{n-1}$ is eompletely separating, let $\delta_{r}=\left(\varnothing, \cdots, \varnothing, \mathscr{D}\left(T_{j}^{r}\right), \varnothing, \cdots, \varnothing\right)$, $r=1,2, \cdots, t, \delta_{t+1}=\delta_{t+2}=\cdots=\delta_{n-1}=(\varnothing, \cdots, \varnothing)$, where $\mathscr{D}\left(T_{j}^{r}\right) \neq \varnothing$ for each $r, r=1,2, \cdots, t$. For each $r, r=1,2, \cdots, t$, each point $x$ of $C$ must satisfy (among other inequalities) the equations $e(S, x)=$ $e(T, x)$, whenever $S, T \in \mathscr{D}\left(T_{j}^{r}\right)$ (see (4.10)). In addition, $\sum_{n=1}^{n} x_{i}=v(N)$. By Lemma 6.5, these equations contain at least $\lambda_{n}$ equations which are linearly independent. Hence, $d(C) \leqq n-\lambda_{n}$.

We shall now give an example of an $n$-person game whose kernel has precisely the dimension $n-\lambda_{n}$. For $n=1,2,3$, any game will do. We therefore assume $n \geqq 4$. First, we construct for each $n, n \geqq 4, \lambda_{n}-1$ sets of coalitions $\mathscr{D}_{n, 1}, \mathscr{D}_{n, 2}, \cdots, \mathscr{D}_{n, \lambda_{n}-1}$ in the following fashion. Each $\mathscr{D}_{n, k}, k=1,2, \cdots, \lambda_{n}-1$, consists of two complementary coalitions. For $n=2^{l}, l=2,3,4, \cdots$, and $k=1,2, \cdots, l$, one divides the ordered sequence $(1,2, \cdots, n)$ into $2^{k}$ equal parts $B_{k, 1}^{(n)}, B_{k, 2}^{(n)}, \cdots, B_{k, 2}^{(n)}$, where $B_{k \mu}^{(n)}$ consists of the players $\left((\mu-1) 2^{l-k}+1,(\mu-1) 2^{l-k}+2, \cdots, \mu 2^{l-k}\right), \mu=1,2, \cdots, 2^{k}$. One coalition in $\mathscr{D}_{n, k}$ is the union $B_{k, 1}^{(n)} \cup B_{k, 3}^{(n)} \cup \cdots \cup B_{k, 2 k-1}^{(n)}$ and the other is the complementary coalition. (See the characteristic vectors for the case $n=8$ ):

$$
\begin{aligned}
& \left.\mathscr{D}_{8,1}^{*}=\left\{\begin{array}{llllllllll}
1 & 1 & 1 & 1 & 0 & 0 & 0 & 0
\end{array}\right),\left(\begin{array}{llllllll}
0 & 0 & 0 & 0 & 1 & 1 & 1 & 1
\end{array}\right)\right\} \\
& \mathscr{D}_{8,2}^{*}=\left\{\left(\begin{array}{lllllllllll}
1 & 1 & 0 & 0 & 1 & 1 & 0 & 0
\end{array}\right),\left(\begin{array}{llllllllll}
0 & 0 & 1 & 1 & 0 & 0 & 1 & 1
\end{array}\right)\right\} \\
& \mathscr{D}_{8,3}^{*}=\left\{\left(\begin{array}{lllllllll}
1 & 0 & 1 & 0 & 1 & 0 & 1 & 0
\end{array}\right), \quad\left(\begin{array}{llllllll}
0 & 1 & 0 & 1 & 0 & 1 & 0 & 1
\end{array}\right)\right.
\end{aligned} .
$$

If $2^{l}<n \leqq 2^{l+1}, \mathscr{D}_{n, k}, k=1,2, \cdots, l+1$ are obtained from $\mathscr{D}_{2^{l+1, k}}$, $k=1,2, \cdots, l+1$, by restricting the coalitions to $n$ players, in such a way that no coalition remains with one player (this is always possible). Define the game $(N ; v)$ by $v(A)=10^{k}+|A| / n$ if $A \in \mathscr{D}_{n, k}, v(N)=1$ and $v(S)=0$, otherwise. Such a choice assures that for every payoff $x, e(A, x)>e(S, x)$ if $A \in \mathscr{D}_{n, k}$ and either $S \in \mathscr{D}_{n, l}, l<k$ or $S \notin \mathscr{D}_{n, l}$. It follows, therefore, that every $x$ which satisfies

$$
x_{i} \geqq 0, i=1,2, \cdots, n ; \sum_{i=1}^{n} x_{i}=1 ; e(A, x)=e(N-A, x)
$$

whenever $A \in \mathscr{D}_{n, k}, k=1,2, \cdots, \lambda_{n}-1$,

generates a profile $P(x)$ (see $\S 4$ ) in which all the sets $F_{j}^{r}$ are empty. Consequently, by Theorem 4.2, the polyhedron (6.10) belongs to the kernel of the game. Since it is determined by $\lambda_{n}$ equations and since it is not empty (it contains the point $(1 / n, 1 / n, \cdots, 1 / n)$ ), its dimension is at least $n-\lambda_{n}$. By the first part of the proof its dimension is at 
most $n-\lambda_{n}$. This completes the proof of the theorem.

THEOREM 6.7. Let $N$ be a set of $n$ players and let $\mathscr{S}=$ $\left\{B_{1}, B_{2}, \cdots, B_{m}\right\}$ be a fixed coalition structure. The maximal dimension of a polyhedron of payoff vectors $x$ such that $(x, \mathscr{B})$ belongs to the kernel of a game is equal to

$$
d(\mathscr{S})=n-\left[\log _{2}\left(\|\mathscr{S}\|-\frac{1}{2}\right)\right]-m-1,
$$

where $\|\mathscr{S}\|=\operatorname{Max}_{1 \leqq j \leqq m}\left|B_{j}\right|$ and $\left|B_{j}\right|$ is the number of the players in the coalition $B_{j}$.

Outline of the proof. Let $d(\mathscr{B})=d(\mathscr{S}, G)=d(C)$, where $C$ is one of the polyhedrons which appear in the representation formula. Using Lemma 6.5 and an inductive procedure similar to the first half of the proof of Theorem 6.6, one can show that the number of linearly independent equations among the equations which are needed to separate the players is at least $\lambda_{\|} \boldsymbol{s} \|-1$; to these equations we have to add the $m$ equations $\sum_{B_{j}} x_{i}=v\left(B_{j}\right), j=1,2, \cdots, m$. Thus, $d(c) \leqq$ $n-\left[\log \left(\|\mathscr{B}\|-\frac{1}{2}\right)\right]-m-1$. An example similar to the one given in Theorem 6.6 shows that equality holds.

7. Improvements in the representation formula. If one wants to use the representation formula (4.14) for actual computation of the kernel of a game, one has to examine an enormous number of potential polyhedra, even for games involving only 4 or 5 players. In practice, however, most of these polyhedra turn out to be empty. For example, the kernels of 3-person games and constant-sum 4-person games consist of a unique point (see [4]). There remains, therefore, the problem of deciding a priori which of the polyhedra in (4.14) are superfluous. The analysis has two aspects: (1) Can one take away polyhedra from (4.14) and still get a representation formula which is valid for all games? (2) Can one deduce information from the characteristic function of a specific class of games to determine $a$ priori that certain polyhedra in (4.14) are superfluous for this specific class? In this section we shall study the first aspect. The second one will be treated in $\$ 10$.

None of the polyhedra in (4.14) can $\alpha$ priori be declared empty, because for a given sequence, $\delta_{1}, \delta_{2}, \cdots, \delta_{n-1}$, generating a profile, it is easy to construct games for which (4.11) is not empty. The simplest game with this property is $(N ; v)$, where $v(S)=0$ for every coalition ${ }^{13}$.

It should be remarked that to each polyhedron which appears in (4.14) there exists a nontrivial pseudo kernel for which this polyhedron

${ }^{13}$ Sometimes this is the only game. Take, for example, the sequence $\delta_{1}=(\{1,23\})$ $\delta_{2}=(\{2,12\})$. 
is not empty ${ }^{14}$.

We shall presently see, however, that many polyhedra can be deleted from (4.14) because they are always contained in other polyhedra.

LEMma 7.1. Let $\delta_{1}, \delta_{2}, \cdots, \delta_{r_{0}}$ be a beginning of a sequence generating a profile. Let

$$
X^{r_{0}}=\bigcap_{r=1}^{r_{0}} X_{1}\left(\delta_{r}, q_{r}\right),
$$

$q_{1}=\{N\}$ (see 4.13), and let $x \in \mathscr{K} \cap X^{r_{0}}$. Under these conditions there exists a continuation $\delta_{r_{0}+1}, \delta_{r_{0}+2}, \cdots, \delta_{n-1}$ such that $x \in \bigcap_{r=1}^{n-1} X_{1}\left(\delta_{r}, q_{r}\right)$.

Proof. $\delta_{1}, \delta_{2}, \cdots, \delta_{r_{0}}$ determine also the part $q_{r_{0}+1}$ of the desired profile. From here continue by the induction process described in (4.4), (4.5) etc. $\delta_{r_{0}+1}(x), \cdots, \delta_{n-1}(x)$ is the desired continuation, since, by (4.10), and (4.13), $x \in \bigcap_{r=r_{0}+1}^{n-1} X_{1}\left(\delta_{r}, q_{r}\right)$.

Lemma 7.2. If $\mathscr{D}\left(T_{j}\right)$ and $\mathscr{D}^{*}\left(T_{j}\right)$ are two sets of coalitions in $\mathscr{C}\left(T_{j}\right)$, if $\mathscr{D}\left(T_{j}\right) \subset \mathscr{D}^{*}\left(T_{j}\right)$, and if $F\left(\mathscr{D}\left(T_{j}\right), T_{j}\right) \subset F\left(\mathscr{D}^{*}\left(T_{j}\right), T_{j}\right)$, then

$$
X_{1}\left(\mathscr{D}\left(T_{j}\right), T_{j}\right) \supset X_{1}\left(\mathscr{D}^{*}\left(T_{j}\right), T_{j}\right) .
$$

The proof follows immediately from (4.10). Combining the two lemmas we obtain the desired result:

Lemma 7.3. With the data used in Lemmas 7.1 and 7.2, $\mathscr{D}^{*}\left(T_{j}\right) \neq$ $\mathscr{D}\left(T_{j}\right)$, let $T_{j} \in q_{r_{0}}$, let $\mathscr{D}\left(T_{j}\right)$ be an element in $\delta_{r_{0}}$, and let $\delta_{r_{0}}^{*}$ be derived from $\delta_{r_{0}}$ by substituting $\mathscr{D}^{*}\left(T_{j}\right)$ for $\mathscr{D}\left(T_{j}\right)$. Under these canditions, one can omit ${ }^{15} \delta_{r_{0}}^{*}$ from $\vartheta\left(q_{r_{0}}\right)$, without violating (4.14).

Proof. By Lemma 7.2, $X^{r_{0}} \supset X^{* r_{0}}$, where

$$
X^{* r_{0}}=\bigcap_{r=1}^{r_{0}-1} X_{1}\left(\delta_{r}, q_{r}\right) \cap X_{1}\left(\delta_{r_{0}}^{*}, q_{r_{0}}\right) .
$$

Consider any sequence $\delta_{1}, \delta_{2}, \cdots, \delta_{r_{0-1}}, \delta_{r_{0}}^{*}, \delta_{r_{0}+1}^{*}, \cdots, \delta_{n-1}^{*}$ generating a profile $P^{*}$. If

$$
x \in \bigcap_{r=1}^{r_{0}-1} X_{1}\left(\delta_{r}, q_{r}\right) \cap X_{1}\left(\delta_{r_{0}}^{*}, q_{r_{0}}^{*}\right) \cap \bigcap_{r=r_{0}+1}^{n-1} X_{1}\left(\delta_{r}^{*}, q_{r}^{*}\right),
$$

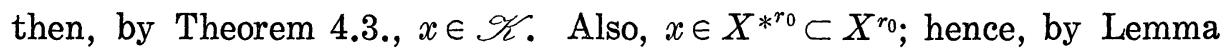
7.1., there exists a sequence $\delta_{1}, \cdots, \delta_{r_{0}}, \cdots, \delta_{n-1}$ generating a profile

${ }^{14}$ See the proof of Theorem 6.6, and the remarks which precede this theorem.

${ }^{15}$ Hence, all the subsequent $q_{r_{0}+1}^{*}, \delta_{r_{0}+1^{\prime}}^{*} s, q_{r_{0}+2^{\prime}}^{*} s, \cdots, \delta_{n-1}^{*} s, q_{n}^{* \prime} s$ which are derived from $\delta_{r_{0}}^{*}$ are also omitted. 
$P$ such that $x \in \bigcap_{r=1}^{n-1} X_{1}\left(\delta_{r}, q_{r}\right)$. Thus, $\delta_{r_{0}}^{*}$ contributes no point to the kernel which cannot be obtained by a different sequence. This completes the proof.

Another obvious short-cut which can be made in the definition of $\vartheta\left(q_{r}\right)$ is to restrict oneself to vectors $\delta_{r}$ which have zero components only when necessary; i.e., for $T_{j}^{r \prime}$ s which contain only single players.

Definition 7.4. A set $\mathscr{D}(T)$ of coalitions of the class $\mathscr{C}(T)$ will be called minimal under separation out if every nonempty proper subset of $\mathscr{D}(T)$ separates out players of $T$, with respect to $T$, which were not separated out by $\mathscr{D}(T)$.

Summarizing the above results, we obtain

THEOREM 7.5. The representation formula (4.14) holds even if one restricts the $\vartheta\left(q_{r}\right)^{\prime}$ s to contain those vectors $\delta_{r}$ which contain only components $\mathscr{D}\left(T_{j}^{r}\right)^{\prime} \mathbf{s}$ which are minimal under separation out and which are nonempty whenever $T_{j}^{r} i s$ not a single player, $r=1,2, \cdots, n-1, j=1,2, \cdots, u_{r}$.

8. Minimal separating sets. We have seen in the last section that it is sufficient to consider in (4.14) sets of coalitions $\mathscr{D}\left(T_{j}^{r}\right)^{\prime} \mathrm{s}$ which are minimal under separation out. This is a significant reduction in the representation formula. For example, if $n=4$, there are $2^{14}-1$ elements in $\vartheta\left(q_{1}\right)$; i.e., 16383 polyhedra that must be examined in the first stage, according to (4.14). Restricting oneself to sets which are minimal under separation out, the number of the polyhedra which need to be examined is 93. It is therefore important both for theoretical and computational purposes to find a procedure which generates the sets which are minimal under separation out. Such procedure should lend itself to computer programming, because the number of such sets increases rapidly with $n$. This section is a contribution in this direction.

Definition 8.1. Let $I$ be a fixed set of players. A collection $\mathscr{D}_{I}$ of subsets ${ }^{16}$ of $I$ is called separating (for the set $I$ ) if $F\left(\mathscr{D}_{I}, I\right)=\varnothing$. It is called minimal separating if it is separating and if no nonempty proper subset of $\mathscr{D}_{I}$ is separating.

LEMMA 8.2. Let $\mathscr{D}(T)$ be a set of coalitions of the class $\mathscr{C}(T)$. Let $I=T-F(\mathscr{D}(T), T)$, and let $\mathscr{D}_{I}$ be a set of subsets of $I$ which is derived from $\mathscr{D}(T)$ by restricting the coalitions of $\mathscr{D}(T)$ to the players in $I$; i.e.,

16 We allow the empty set to be an element of $\mathscr{D}_{I}$. 


$$
\mathscr{D}_{I}=\{A \cap I: A \in \mathscr{D}(T)\} \text {. }
$$

With this notation, $\mathscr{D}(T)$ is minimal under separation out if and only if

(i) $\mathscr{D}_{I}$ is minimal separating,

(ii) $A, B \in \mathscr{D}(T), A \neq B \Rightarrow A \cap I \neq B \cap I$.

Proof. Suppose (i) and (ii) are satisfied. Let $\mathscr{D}^{*}(T)$ be a nonempty proper subset of $\mathscr{D}(T)$. Restricting the coalitions of $\mathscr{D}^{*}(T)$ to the players in $I$, we obtain a nonempty proper subset $\mathscr{D}_{I}{ }^{*}$ of $\mathscr{D}_{I}$. By (i), a player $l, l \in I$, is separated out by $\mathscr{D}_{I}{ }^{*}$, with respect to $I$; hence $l$ is separated out by $\mathscr{D}^{*}(T)$ with respect to $T$. Consequently, $\mathscr{D}(T)$ is minimal under separation out.

Conversely, let $\mathscr{D}(T)$ be minimal under separation out. This means that for every nonempty proper subset $\mathscr{D}^{*}(T)$ of $\mathscr{D}(T)$ there exists a player $l, l \in I$, who is separated out by $\mathscr{D}^{*}(T)$, with respect to $T$. Moreover, there must be a player $k$ in $I$ who separates player $l$ out by $\mathscr{D}^{*}(T)$. Indeed, if $k \in T-I$ and $k$ separates $l$ out by $\mathscr{D}^{*}(T)$, then $k \in F(\mathscr{D}(T), T)$ and there exists a player $k_{0}, k_{0} \in I$ who has separated player $k$ out by $\mathscr{D}(T)$ (see part iv of Lemma 3.1). Clearly, $k_{0}$ also separates $l$ by $\mathscr{D}^{*}(T)$.

If (ii) is not satisfied, there exist coalitions $A, B$ in $\mathscr{D}(T), A \neq B$, such that $A \cap I=B \cap I$. Consider the set $\mathscr{D}^{*}(T)=\mathscr{D}(T)-\{B\}$; then its restriction to the players in $I$ yields again the set $\mathscr{D}_{I}$. We have seen that there exists a player $k$ in $I$ who separates a player $l$ out by $\mathscr{D}^{*}(T), l \in I$; consequently, $F\left(\mathscr{D}_{I}, I\right) \neq \varnothing$. This is impossible because, by the definition of $I, \mathscr{D}_{I}$ is separating.

We shall show that $\mathscr{D}_{I}$ is minimal separating. Indeed, every nonempty proper subset $\mathscr{D}_{I}^{* *}$ of $\mathscr{D}_{I}$ is generated from a nonempty proper subset $\mathscr{D}^{* *}(T)$ of $\mathscr{D}(T)$ by restriction to the players of $I$, and, as before, we obtain $F\left(\mathscr{D}_{I}{ }^{* *}, I\right) \neq \varnothing$.

There remains the problem of constructing all the minimal separating collections for subsets $I$ of $T$. For each such set $\mathscr{D}_{I}$ one adds the players of $T-I$ in all possible ways to the coalitions of $\mathscr{D}_{I}$ so as to leave them separated out by members of $I$. This is easily done, at least theoretically.

Actually, it suffices to construct all the minimal completely separating collections, i.e., collections which are minimal under the property that every two players separate each other by them (see $\S 6$ ). Minimal separating collections are then obtained by deleting collections which are not minimal separating ${ }^{17}$ and adding players to the coalitions in the remaining collections in such a way that each new player and a

17 E.g., $\{\{12\},\{13\},\{24\},\{34\}\}$ is a minimal completely separating collection which is not minimal separating, because $\{\{12\},\{34\}\}$ is a separating collection. 
previous player are inseparable.

The following lemma serves to construct the completely separating collections.

LEMMA 8.3. Let $I=\{1,2, \cdots, m\}, \bar{I}=\{1,2, \cdots, m, m+1\} . \quad$ Let $\overline{\mathscr{D}}$ be a collection of subsets of $\bar{I}$. Denote

$$
\begin{aligned}
& \mathscr{D}=\{S: S=\bar{S} \cap I, \bar{S} \in \overline{\mathscr{D}}\}, \\
& \mathscr{R}_{1}=\{S: S \in \overline{\mathscr{D}} \text { and } S \subset I\}, \\
& \mathscr{R}_{2}=\{S: S \subset I \text { and } S \cup\{m+1\} \in \overline{\mathscr{D}}) .
\end{aligned}
$$

with this notation, $\overline{\mathscr{D}}$ is a completely separating collection for $\bar{I}$ if and only if $\mathscr{D}$ is a completely separating collection for $I$ and

$$
\cup\left\{S: S \in \mathscr{R}_{1}\right\}=I, \quad \cap\left\{S: S \in \mathscr{R}_{2}\right\}=\varnothing .
$$

Proof. If $\overline{\mathscr{D}}$ is completely separating collection for $I$, then clearly so is $\mathscr{D}$. Moreover, the first relation of (8.5) holds, because $m+1$ separates no player $k$ in $I$ out by $\overline{\mathscr{D}}$, nor are $m+1$ and $k$ inseparable by $\overline{\mathscr{D}}$. Similarly, the second relation holds, because $m+1$ is not separated out by $\overline{\mathscr{D}}$. Conversely, if $\mathscr{D}$ is completely separating and (8.5) holds, then player $m+1$ neither separates any player $k$ in $I$ out by $\overline{\mathscr{D}}$, nor are $m+1$ and $k$ inseparable by $\overline{\mathscr{D}}$. Moreover, by the second relation in (8.5), player $m+1$ is not separated out; hence $\overline{\mathscr{D}}$ is completely separating.

9. Desirability relations. Two players $k$ and $l$ are called symmetric in a game $(N ; v)$, if the game remains invariant when $k$ and $l$ exchange roles, i.e., if

$$
v(S \cup\{k\})=v(S \cup\{l\}), \quad \text { whenever } \quad k, l \notin S .
$$

This symmetry notion proved to be a useful criterion in checking the merits of various solution concepts for $n$-person games; for it has been felt intuitively that any kind of a solution (or, at least, the set of solutions) should not distinguish between symmetric players.

An immediate generalization of invariance under symmetry, is invariance under a group of permutations. Another possibility would be to treat symmetric players as equal and define appropriately order relations among the players. This line will be pursued in this section.

Definition 9.1. A player $k$ is said to be more desirable than a player $l$ in a game $G=(N ; v)$, and this is denoted by $k \varepsilon l$, if

$$
v(S \cup\{k\}) \geqq v(S \cup\{l\}), \quad \text { whenever } \quad k, l \notin S .
$$


This definition generalizes Isbell's notion of replacement, ([6] p. 428). Intuitively, if $k £ l$, and $k, l \notin S$, then $S$ will prefer $k$ to $l$ as an additional partner.

THEOREM 9.2. Desirability in a game is a reflexive and transitive relation.

Proof. Obviously $\Xi$ is a reflexive relation. To prove transitivity, let $k £ l, l £ m$; we may assume $k \neq m$. Let $S$ be an arbitrary coalition such that $k, m \notin S$. If $l \notin S$, then $v(S \cup\{k\}) \geqq v(S \cup\{l\}) \geqq$ $v(S \cup\{m\})$. If $l \in S$, letting $S_{1}=S-\{l\}$, we then have $v(S \cup\{k\})=$ $v\left(S_{1} \cup\{k\} \cup\{l\}\right) \geqq v\left(S_{1} \cup\{k\} \cup\{m\}\right) \geqq v\left(S_{1} \cup\{l\} \cup\{m\}\right)=v(S \cup\{m\})$. Thus, $k \succsim m$.

Clearly, $k$ and $l$ are symmetric if and only if $k \xi l$ and $l \xi k$. In this case we shall write $k \sim l$.

We say that a vector $x=\left(x_{1}, \cdots, x_{n}\right)$ preserves the desirability relation with respect to a game $G$, if $k £ l$ implies $x_{k} \geqq x_{l}$.

THEOREM 9.3. The desirability relation is preserved by the kernel ${ }^{18}$.

Proof. Let $x \in \mathscr{K}$. Suppose that $k £ l$, but $x_{k}<x_{l}$. Let $S$ be a coalition in which $l$ attains maximum excess against $k$; i.e., $s_{l, k}=e(S)$, $S \in \mathscr{T}_{l, k}$. Let $T=(S \cup\{k\})-\{l\}$; then $v(T) \geqq v(S)$. It follows that $e(T)>e(S)$, because $x_{l}>x_{k}$. Thus $s_{k, l}>s_{l, k}$ and $x_{l}>x_{k} \geqq 0$; hence $k \gg l$, and $x$ cannot be in the kernel.

For a general coalition structure, Theorem 9.3 generalizes as follows:

THEOREM 9.3a. Let $G$ be an n-person game, and let $\mathscr{B}$ be a coalition structure in $G$. Let $(x ; \mathscr{B}) \in \mathscr{K}(G)$, and let $k$ and $l$ be two members of the same coalition in $\mathscr{B}$, such that $k$ is more desirable than $l$, then $x_{k} \geqq x_{l}$.

A player $l$ in a game $G=(N ; v)$ is called a dummy if $v(S \cup\{l\}) \leqq$ $v(S)$ for all the coalitions $S$. Let $x$ be an arbitrary payoff vector in $G$, and let $\mathscr{D}=\mathscr{D}(N, x)$ be the set of coalitions having maximum excess among the coalitions of $\mathscr{C}(N)$. Then, a dummy player $l$ who receives a positive payment in $x$ can be in no coalition of $\mathscr{D}$ which contains more than one player, because $e(S \cup\{l\}, x)<e(S, x)$ (see (2.5)). We shall show that also the 1-person coalition $\{l\}$ cannot be in $\mathscr{D}$. Indeed, $v(N-\{l\}) \geqq v(N)$, because $l$ is a dummy player; hence, $e(N-\{l\}, x)>e(N, x)=0$, because $x_{l}>0$. Therefore, the coalitions

${ }^{18}$ Namely, it is preserved by $x$ if $(x, N) \in \mathscr{K}$. See the end of Section 2. 
of $\mathscr{D}$ have a positive excess, whereas $e(\{l\}, x)=-x_{l}<0$. We have proved that $\mathscr{D}$ separates such a player out. Combining this result with Theorem 4.2 we obtain:

Theorem 9.4. Dummy players always obtain a zero payment in the kernel.

Let us now say that $k$ is strictly more desirable than $l,(k \in l)$, if $k £ l$ but not $l \equiv k$. Clearly, $\varepsilon$ is a partial order, and one may ask if the kernel strictly preserves desirability, i.e., if $k \varepsilon l$ implies $x_{k}>x_{l}$, for $x \in \mathscr{K}$. Unfortunately, the answer is negative:

EXAMPLE 9.5. Consider the 3-person game $(123 ; v)$ where $v(12)=$ $v(13)=1, v(123)=3, v(S)=0$ otherwise. In this game $1 \varepsilon 2,1 \varepsilon 3$, yet the kernel consists of the unique payoff vector $(1,1,1)$.

Although we have seen that strict desirability is of little value in the kernel theory, it may be applied in other aspects of $n$-person game theory. For instance, it is easy to see that the Shapley value (see [11]) strictly preserves desirability. Another interesting application is found in the representation of weighted majority games:

EXAMPLE 9.6. Let $G=\left[q ; w_{1}, w_{2}, \cdots, w_{n}\right]$ be a weighted majority game $^{19}$. Clearly, $w_{k} \geqq w_{l}$ implies $k £ l$, and therefore, the desirability relation is complete. Focusing our attention on strict desirability we can still say that $w_{k}=w_{l}$ implies $k \sim l$; however, it is not true, in general, that $w_{l}>w_{l}$ implies $k \varepsilon l$; because the weights of $G$ are not uniquely determined. One can only claim the following.

THEOREM 9.7. A weighted majority game $G$ can be represented by weights that strictly preserve the desirability relation ${ }^{20}$.

Proof. Let $S_{1}, S_{2}, \cdots, S_{k}$ be the equivalence classes of the symmetric players in $G$. Denote by $s_{j}$ the number of players of $S_{j}, j=1,2, \cdots, k$. For $k \in S_{j}$, define $w_{k}^{*}=\left(1 / s_{j}\right) \sum_{\nu \in S_{j}} w_{\nu}$, where $\left[q ; w_{1}, w_{2}, \cdots, w_{n}\right]$ is any

${ }^{19}$ We adopt the notation used by L. S. Shapley [10]. With this notation, a coalition $S$ is winning if and only if $\sum_{i \in S} w_{i} \geqq q$.

20 One may hope that a simple game in which the desirability relation is complete must be a weighted majority game. A counter example to such a conjecture has been provided by R. J. Aumann: Consider an 8-person simple game, in which the winning coalitions are $\{1458\},\{2367\}$, all the coalitions which result from these if one wants that $1 £ 2 £ \cdots \S 8$ will hold, and their supersets. If this game is a weighted majority game, then the sum of the weights is greater than or equal to $2 q$ because the game contains two disjoint 4-person winning coalitions. But the game contains also the two losing complementary coalitions, $\{1278\}$ and $\{3456\}$, hence the same sum is less than $2 q$, which obviously is impossible. 
particular representation of $G$. Then, $\left[q ; w_{1}^{*}, w_{2}^{*}, \cdots, w_{n}^{*}\right]$ is the desired representation.

REMARK. It is easy to see that the homogeneous weights of constant-sum homogeneous weighted majority games (see J. von Neumann and 0 . Morgenstern [13]) strictly preserve the desirability relations.

The excess of a coalition (see (2.5)) plays a decisive role in the kernel theory. In particular, one wants to be able to compare, if possible, excesses of various coalitions with respect to the payoff vectors of the kernel, without actually computing the kernel. To achieve this we introduce:

Definition 9.8. Let $A$ and $B$ be two coalitions in a game $G$. We say that $A$ is wealthier than $B$ in $G$, and denote this by $A \geqq B$, if

$$
v(B) \geqq v(A),
$$

and if, in addition, there exists a one-to-one mapping $\lambda$ of a subcoalition $A_{1}, A_{1} \subset A$, onto $B$, such that

$$
i £ \lambda(i) \text {, for each } i, i \in A_{1} \text {. }
$$

Clearly the wealth relation is reflexive and transitive. By Theorem 9.3 and (9.4) it follows that if $A \geqq B$, then, in spite of the fact that $v(B) \geqq v(A)$, for every payoff vector in the kernel of $G$, the total poyoff received by the players of $A$ is not less than that received by $B$. In view of (2.5) and (9.3) one finds that $e(B, x) \geqq e(A, x)$ for $x \in \mathscr{K}$. Thus we have proved:

THEOREM 9.9. A wealthier of two coalitions has the smaller excess, in the weak sense, for every payoff vector in the kernel.

Just as in the case of desirability, we can say that $A$ is strictly wealthier than $B(A>B)$ if $A \geqq B$ and $B \supsetneqq A$; and that $A$ and $B$ are equally wealthy $(A \cong B)$ if $A \geqq B$ and $B \geqq A$. Both relations are transitive, and, furthermore, they seem to be natural because of the following:

THEOREM 9.10. If $A \cong B$ then $v(A)=v(B)$, and the mappings which satisfy (9.4) must be functions which map players onto symmetric ones.

Proof. Clearly, $v(A)=v(B)$ and $A$ and $B$ have the same number of players. Let $\lambda$ be a one-to-one mapping of $A$ onto $B$ such that $i £ \lambda(i)$ for each $i, i \in A$. Similarly, let $\mu$ be a one-to-one mapping 
of $B$ onto $A$ such that $j £ \mu(j)$ for each $j, j \in B$; then $\nu=\mu \lambda$, is a mapping of $A$ onto itself such that $i £ \nu(i)$ for each $i, i \in A$.

In view of the finite number of players in $A$, there exists an orbit $i £ \nu(i) £ \nu^{2}(i) £ \cdots \equiv \nu^{k}(i)$, where $\nu^{k}(i)=i$; therefore, $i \sim \nu(i)$ for each $i$. Thus, $i \sim \lambda(i)$ for each $i$ in $A$ and $j \sim \mu(j)$ for each $j$ in $B$. This completes the proof.

10. Information on the characteristic function which simplifies the representation formula. Even with the simplification gained in $\$ 7$, the representation formula contains too many polyhedra to make a joyful task of the computation by hand of the kernel of, say, a 6-person game. In this section we shall show how knowledge of the characteristic function, and, in particular, of the desirability relations may be used to determine a priori polyhedra which need not be computed-either because they are empty or because they are contained in others.

Lemma 10.1. Consider the game $G=(N ; v)$ and let

$$
X(N ; \varepsilon)=\left\{x: x \in X(N), x_{l} \geqq x_{l} \text { whenever } k £ l\right\} \text {, }
$$

then, Theorem 7.5 remains true if

(i) $X_{1}\left(\mathscr{D}\left(T_{j}^{r}\right), T_{j}^{r}\right)$ is replaced by

$$
X_{2}\left(\mathscr{D}\left(T_{j}^{r}\right), T_{j}^{r}\right)=X_{1}\left(\mathscr{D}\left(T_{j}^{r}\right), T_{j}^{r}\right) \cap X(N ; \varepsilon),
$$

for each $r$ and $j$.

(ii) $\mathscr{D}\left(T_{j}^{r}\right)$ is taken to be the empty set whenever $T_{j}^{r}$ contains only symmetric players.

Proof. By Theorem 9.3, $\mathscr{K}(G) \subset X(N$; $\leftleftarrows)$; therefore each of the polyhedra which compose $\mathscr{K}$ is contained in $X(N$; $\Sigma)$; hence, making the replacement (i) leaves $\bigcap_{r=1}^{n-1} X_{1}\left(\delta_{r}, q_{r}\right)$ unchanged; i.e.,

$$
\bigcap_{r=1}^{n-1} X_{1}\left(\delta_{r}, q_{r}\right)=\bigcap_{r=1}^{n-1} X_{2}\left(\delta_{r}, q_{r}\right)
$$

where

$$
X_{2}\left(\delta_{r}, q_{r}\right)=X_{1}\left(\delta_{r}, q_{r}\right) \cap X(N ; \xi) .
$$

Restricting the sets $\mathscr{D}\left(T_{j}^{r}\right)$ as described by (ii) means that symmetric players were left unseparated in the profile generated by $\delta_{1}, \cdots, \delta_{n-1}$. Consequently, $\mathrm{\cap}_{r=1}^{n-1} X_{2}\left(\delta_{r}, q_{r}\right)$ might, perhaps ${ }^{21}$, contain points which are not in $\mathscr{K}$. This is not the case, since nonsymmetric players are

\footnotetext{
${ }^{21}$ There may be now fewer proper intersections.
} 
balanced by the construction, and symmetric players are balanced because they receive equal payoffs due to the fact that the payoff vectors belongs to $X(N ; \varepsilon)$. This completes the proof.

LeMma 10.2. Let $T$ be a subset of $N$ and let $\mathscr{D}(T)$ be a collection of coalitions of $\mathscr{C}(T)$. In order that $X_{1}(\mathscr{D}(T), T)$ be nonempty, it is necessary that

(i) for each coalition $A$ in $\mathscr{D}(T)$ there exists no coalition $B$ in $\mathscr{C}(T)$ such that $v(B)>v(A)$ and such that the players of $B-A$, if such exist, are separated out by $\mathscr{D}(T)$, with respect to $T$; i.e., $B-A \subset F(\mathscr{D}(T), T)$;

(ii) either $v\left(A_{1} \cup A_{2}\right) \leqq v\left(A_{1}\right)+v\left(A_{2}\right)$, or $v\left(A_{1} \cup A_{2}\right) \leqq v(N)$, whenever $A_{1}, A_{2}$ are disjoint coalitions in $\mathscr{D}(T)$ and $A_{1} \cup A_{2}$ belongs to $\mathscr{C}(T)$.

Proof. Clearly, if condition (i) is violated, then $X_{1}(\mathscr{D}(T), T)$ is empty (see (4.10)), because $x_{i}=0$ for $i \in B-A$ implies $e(B, x)>e(A, x)$, contrary to the maximality of $e(A, x)$.

If condition (ii) is violated, then, there exist two disjoint coalitions $A_{1}$ and $A_{2}$ in $\mathscr{D}(T)$ such that $A_{1} \cup A_{2} \in \mathscr{C}(T), v\left(A_{1} \cup A_{2}\right)>v(N)$ and $v\left(A_{1} \cup A_{2}\right)>v\left(A_{1}\right)+v\left(A_{2}\right)$. If $x \in X_{1}(\mathscr{D}(T), T)$, then $e\left(A_{1} \cup A_{2}, x\right)>e(N, x)=$ 0 and

$$
e\left(A_{1} \cup A_{2}, x\right)>e\left(A_{1}, x\right)+e\left(A_{2}, x\right)=2 e\left(A_{1}, x\right) ;
$$

consequently, $e\left(A_{1} \cup A_{2}, x\right)>e\left(A_{1}, x\right)$, contrary to the maximality of $e\left(A_{1}, x\right)$. This again shows that $X_{1}(\mathscr{D}(T), T)$ is empty.

We shall now pass to short cuts which can be deduced from the desirability relations which may occur in a game. This is motivated by Theorem 9.9 from which it follows that if $x \in \mathscr{K}$, if $A \in \mathscr{D}(T, x)$ and if $A$ is wealthier than $B$, where $B$ is a coalition in $\mathscr{C}(T)$, then $B \in \mathscr{D}(T, x)$.

Definition 10.3. A set $\mathscr{D}(T)$ of coalitions of the class $\mathscr{C}(T)$ will be called saturated (with respect to $\mathscr{C}(T)$ ), if

$$
A \in \mathscr{D}(T), B \in \mathscr{C}(T), A \geqq B \text { implies } B \in \mathscr{D}(T) \text {. }
$$

THEOREM 10.4. The representation formula (4.14) remains true if the following modification are made:

(i) The sets $\vartheta\left(q_{r}\right), r=1,2, \cdots, n-1$, are restricted to contain only $\delta_{r}{ }^{\prime} \mathrm{s}$ whose components are empty for $T_{j}^{r \prime} \mathrm{s}$ which contain only single or symmetric players and otherwise are saturated ${ }^{22}$.

${ }^{22}$ Note that such a restriction on a $\vartheta\left(q_{r}\right)$ implies a restriction on the subsequent possible $q_{r+1}$ 's $q_{r+2}$ 's etc. 
(ii) $X_{1}\left(\mathscr{D}\left(T_{j}^{r}\right), T_{j}^{r}\right)$ is replaced by $X_{2}\left(\mathscr{D}\left(T_{j}^{r}\right), T_{j}^{r}\right)$ (see (10.2)) for each $r$ and $j$.

Proof. Clearly, the resulting left hand side expression of (4.14) subject to the above modifications is contained in $\mathscr{K}$ (see the proof of Lemma 10.1).

To show the reverse inclusion let $x \in \mathscr{K}$. We shall define a profile $P^{*}(x)$ in a way quite similar to the profile $P(x)$ which has been defined at the beginning of $\S 4$ :

Starting with $q_{1}^{*}=\{N\}, \delta_{1}^{*}(x)=\mathscr{D}(N, x)$ (see (4.2)), suppose we have arrived at a $q_{r}^{*}(x)=\left\{T_{1}^{r}, T_{2}^{r}, \cdots, T_{u_{r}}^{r}\right\}$. For each $T_{j}^{r}$ which consists neither of a single player nor of symmetric players, let $\mathscr{D}\left(T_{j}^{r}, x\right)$ be the set of coalitions in $\mathscr{C}\left(T_{j}^{r}\right)$ which obtain a maximum excess (with respect to $\mathscr{C}\left(T_{j}^{r}\right)$ ). These sets are saturated (with respect to $\mathscr{C}\left(T_{j}^{r}\right)$ ). If $T_{j}^{r}$ consists either of a single player or of symmetric players, we define $\mathscr{D}\left(T_{j}^{r}, x\right)$ bo be the empty set. Let

$$
\delta_{r}^{*}(x)=\left(\mathscr{D}\left(T_{1}^{r}, x\right), \cdots, \mathscr{D}\left(T_{u_{r}}^{r}, x\right)\right),
$$

then $q_{r+1}^{*}$ is defined in the usual fashion. Clearly, $\delta_{1}^{*}(x), \delta_{2}^{*}(x), \cdots, \delta_{n-1}^{*}(x)$ satisfy all the restrictions of the theorem and $x \in \bigcap_{r=1}^{n-1} X_{2}\left(\delta_{r}^{*}, q_{r}^{*}\right)$.

EXAMPLE 10.5. Consider the 4-person game $(1234 ; v)$, where $v(14)=v(24)=v(34)=v(23)=v(1234)=1, v(S)=0$ otherwise. Clearly, for every payoff vector $x$, the maximum excess is positive and is therefore attained only by coalitions of positive value. Thus, only such coalitions are candidates for $\mathscr{D}(N)$, since, otherwise $X_{2}(\mathscr{D}(N), N)=\varnothing$. Moreover, since $1-32 \sim 3-34$, which implies $\{24\} \cong\{34\} \geqq\{14\}$ and $\{24\} \cong\{34\} \geq\{23\}$, it follows that a saturated $\mathscr{D}(N)$ must contain either $\{14\}$ or $\{23\}$, and it contains both coalitions if it contains at least two coalitions. But if $\mathscr{D}(N)$ consists of one of these coalitions, then again $X_{2}(\mathscr{D}(N), N)=\varnothing$. Consequently, a saturated $\mathscr{D}(N)$ is either $\mathscr{D}_{1}=\{\{14\},\{23\}\}$ or $\mathscr{D}_{2}=\{\{14\},\{23\},\{24\},\{34\}\}$. We need not consider the second case, because $\mathscr{D}_{1} \subset \mathscr{D}_{2}$ and $F\left(\mathscr{D}_{1}, N\right) \subset F\left(\mathscr{D}_{2}, N\right)$. (See the proof of Theorem 7.5 and the remarks which follow this example.) Thus, $\delta_{1}\left(q_{1}\right)=(\{\{14\},\{23\}\})$. There is no need to further separate between player 2 and player 3 , because $2 \sim 3$. Consequently, $\delta_{2}=\mathscr{D}(14)$ has one component which consists of coalitions of the class $\mathscr{C}(14)$. The only saturated possibility which gives rise to a nonempty polyhedron is $\delta_{2}=(\mathscr{D}(14))=(\{\{24\},\{34\}\})$. We conclude that $\mathscr{K}=X_{2}\left(\delta_{1}, 1234\right) \cap X_{2}\left(\delta_{2}, 14\right)$, which is the unique point $(0,1 / 4,1 / 4,1 / 2)$.

Theorem 7.5 (and Lemma 10.1) suggest improvements by restricting the $\mathscr{D}\left(T_{j}^{r}\right)^{\prime}$ s to be minimal under separation out. Theorem 10.4 suggests improvements by considering only saturated $\mathscr{D}\left(T_{j}^{r}\right)^{\prime} \mathrm{s}$. One should 
be careful, however when one applies both theorems simultaneously. Take, for example, the 4-person game $(1234 ; v)$, where

$$
v(12)=v(13)=v(24)=v(34)=v(1234)=1, v(S)=0
$$

otherwise. Here $(1 / 4,1 / 4,1 / 4,1 / 4) \in \mathscr{K}$. It is obtained as a point in $X_{2}(\mathscr{D}(N), N)$ only for a $\mathscr{D}(N)$ which is $\{\{13\},\{24\}\}$ or $\{\{12\},\{34\}\}$ or $\{\{12\},\{13\},\{24\},\{34\}\}$. The first two sets are minimal under separation out but are not saturated, whereas the last set is saturated but it is not minimal under separation out. Thus, if one restricts $\mathscr{D}(N)$ to be both minimal under separation out and saturated, one will never obtain $(1 / 4,1 / 4,1 / 4,1 / 4)$. The correct procedure would be either of the following two:

(i) For a given $T_{j}^{r}$ consider all sets $\mathscr{D}\left(T_{j}^{r}\right)$ which are minimal under separation out and delete from them nonsaturated ones provided that by adding less wealthy coalitions you reach a set which is minimal under separation out.

(ii) For a given $T_{j}^{r}$, consider all the saturated sets $\mathscr{D}\left(T_{j}^{r}\right)$, and delete from them sets which are not minimal under separation out provided that for each set $\mathscr{D}^{*}\left(T_{j}^{r}\right)$ which you delete there exists a saturated set $\mathscr{D}^{* *}\left(T_{j}^{r}\right)$ such that $\mathscr{D}^{* *}\left(T_{j}^{r}\right) \subset \mathscr{D}^{*}\left(T_{j}^{r}\right)$ and $F\left(\mathscr{D}^{* *}\left(T_{j}^{r}\right), T_{j}^{r}\right) \subset F\left(\mathscr{D}^{*}\left(T_{j}^{r}\right), T_{j}^{r}\right)$.

EXAMPLE 10.6. Consider the 6-person weighted majority game $[8 ; 5,4,2,2,1,1]$. We shall illustrate our method by computing its kernel.

Let $x \in X(N$; $\leftleftarrows)$ (see 10.1), then, by Example 9.6, $x_{1} \geqq x_{2} \geqq x_{3}=$ $x_{4} \geqq x_{5}=x_{6}$. Obviously, $(1,0,0,0,0,0) \notin \mathscr{K} \quad$ (because $s_{1,2}(x)<s_{2,1}(x)$ with respect to this payoff vector). Therefore, in order to apply Theorem 10.4, it is sufficient to restrict oneself to payoff vectors for which $x_{1}, x_{2}>0$. In other words, both players 1 and 2 must not be separated out at any stage (see (4.10), (4.13) and (4.14)).

Obviously, the maximum excess $s_{1}(x)$ (see (4.3)) of any payoff vector must be obtained at some minimal winning coalition, and $s_{1}(x)>0$. Consequently, no losing coalition is in $\mathscr{D}(N)=\mathscr{D}\left(q_{1}\right)$. Moreover, if $s_{1}(x)$ is obtained in a winning coalition $S$ which is not a minimal winning coalition and if $T$ is a minimal subcoalition of $S$, then the members of $S-T$ must obtain a zero payment in $x$.

Since 1 and 2 are not separated out, them two possibilities may occur: (i) $\mathscr{D}(N)$ contains the coalition $\{12\}$ and does not intersect both $\mathscr{T}_{1,2}$ and $\mathscr{T}_{2,1}$ (see $(2.6)$ ). By the previous remarks, and since players who are separated out receive a zero payment in $X_{2}(\mathscr{D}(N), N)$, it follows that $x_{3}=x_{4}=x_{5}=x_{6}=0$ for $x \in X_{2}(\mathscr{D}(N), N)$. Consequently, $s_{1}(x)=0$, which is impossible. (ii) $\mathscr{D}(N)$ intersects either $\mathscr{T}_{1,2}$ or 
$\mathscr{T}_{2,1}$ and therefore it intersects both sets. Examinning the minimal winning coalitions and taking into account that $x \in X(N ; \xi)$, we find that in order that $X_{2}(\mathscr{D}(N), N) \neq \varnothing, \mathscr{D}(N) \cap \mathscr{T}_{1,2}$ must contain the set $\mathscr{D}_{1} \equiv\{\{135\},\{136\},\{145\},\{146\}\}$ and $\mathscr{D}(N) \cap \mathscr{T}_{2,1}$ must contain at least one of the following sets: $\mathscr{D}_{2} \equiv\{\{234\}\}$ or $\mathscr{D}_{3} \equiv\{\{2356\},\{2456\}\}$.

Clearly, $\mathscr{D}(N) \neq \mathscr{D}_{1} \cup \mathscr{D}_{2} \equiv \mathscr{D}_{4}$ and $\mathscr{D}(N) \neq \mathscr{D}_{1} \cup \mathscr{D}_{3} \equiv \mathscr{D}_{5}$, because in both cases player 2 is separated out; therefore $\mathscr{D}(N) \supset \mathscr{D}_{4} \cup\{12\} \equiv \mathscr{D}_{6}$ or $\mathscr{D}(N) \supset \mathscr{D}_{5} \cup\{12\} \equiv \mathscr{D}_{3}$ or $\mathscr{D}(N) \supset \mathscr{D}_{4} \cup \mathscr{D}_{5} \equiv \mathscr{D}_{8}$.

Suppose $\mathscr{D}(N)=\mathscr{D}_{6}$, then players 5 and 6 are separated out and $x_{5}=x_{6}=0$. Now, for $x \in X_{2}\left(\mathscr{D}_{6}, N\right), s_{1}(x)=e(\{234\}, x) \geqq e(\{2356\}, x)$; hence $x_{4}=x_{3}=0$, and, clearly, $e(\{12\}, x)$ cannot have a maximum excess. Thus, $X_{2}\left(\mathscr{D}_{6}, N\right)=\varnothing$. Similarly, if $\mathscr{D}(N) \supset \mathscr{D}_{6}$, then $\mathscr{D}(N)$ must contain other coalitions such that players 5 and 6 may obtain a positive payment in $X_{2}(\mathscr{D}(N), N)$. This is possible only if, in addition, $\mathscr{D}(N) \supset \mathscr{D}_{7}$. We are therefore left with the two possibilities: $\mathscr{D}(N) \supset \mathscr{D}_{7}$ and $\mathscr{D}(N) \supset \mathscr{D}_{8}$.

Clearly, $\mathscr{D}_{7}$ and $\mathscr{D}_{8}$ are completely separating, saturated sets (see Definitions 6.1 and 10.3) and every set containing any one of them is completely separating. By Lemma 7.3 (see also Theorem 7.5), it is enough to consider only $\mathscr{D}_{7}$ and $\mathscr{D}_{8}$ as candidates of $\mathscr{D}(N)$. Therefore $\mathscr{K}=X_{2}\left(\mathscr{D}_{7}, N\right) \cup X_{2}\left(\mathscr{D}_{8}, N\right)=[(4 / 13,3 / 13,2 / 13,2 / 13,1 / 13,1 / 13)(1 / 4$, $1 / 4,1 / 4,0,0,0)] \cup[(4 / 13,3 / 13,2 / 13,2 / 13,1 / 13,1 / 13)(1 / 2,1 / 2,0,0,0,0)]$. This is a $V$-shaped configuration.

EXAmple 10.7. Consider a 5-person game, whose characteristic function satisfies: $v(25)=v(35)=v(45)=1, v(123)=11 / 12, v(S)=1$ for all the remaining 3 and 4-person coalitions, $v(12345)=2$ and $v(S)=0$ otherwise. We shall compute the kernel of this game.

Clearly, $x_{1} \leqq x_{2}=x_{3} \leqq x_{4} \leqq x_{5}$, because $1 \geqq 2 \sim 3 \geqq 4$ ․ Also, $(0,0,0, \alpha, 2-\alpha) \notin \mathscr{K}$, because $s_{1,5}=11 / 12>0$ and $s_{5,1} \leqq 0$ with respect to this payoff vector. Consequently, $x_{2}, x_{3}, x_{4}, x_{5}>0$ for every payoff vector in $\mathscr{L}$, and we can restrict ourselves to collections for which at most player 1 is separated out.

For each payoff vector $x, s_{1}(x) \geqq 0$; therefore the maximum excess must be obtained in coalitions from the set: $\{\{25\},\{35\},\{45\},\{124\}$, $\{134\},\{234\},\{123\}\}$. By saturation

$$
\begin{aligned}
\{45\} \in \mathscr{D}(N) & \Rightarrow\{25\},\{35\} \in \mathscr{D}(N), \\
\{234\} \in \mathscr{D}(N) & \Rightarrow\{124\},\{134\} \in \mathscr{D}(N), \\
\{25\} \in \mathscr{D}(N) & \Leftrightarrow\{35\} \in \mathscr{D}(N), \\
\{124\} \in \mathscr{D}(N) & \Leftrightarrow\{134\} \in \mathscr{D}(N) .
\end{aligned}
$$

A saturated $\mathscr{D}(N)$ which does not contain $\{124\}$ implies $x_{4}=0$ for $x \in X_{2}(\mathscr{S}(N), N)$, because player 4 is separated out by such set. 
Similarly, a saturated $\mathscr{D}(N)$ which does not contain $\{25\}$ implies $x_{5}=0$ for $x \in X_{2}(\mathscr{D}(N), N)$. Therefore $\mathscr{D}(N) \supset\{\{25\},\{35\},\{124\},\{134\}\} \equiv \mathscr{D}_{1}$. Since $\mathscr{D}_{1}$ is a separating saturated set, we have to consider only the case $\mathscr{D}(N)=\mathscr{D}_{1}$ in the first stage. In this case

$$
X_{2}(\mathscr{D}(N), N)=\left\{x \mid x \in X(N ; \xi), x_{5}=x_{1}+x_{4}, x_{1}+2 x_{2} \geqq 11 / 12\right\}
$$

and players 1 and 4 have to be separated in the next stage.

It should be noted that $s_{1}(x)=0$ for $x \in X_{2}(\mathscr{D}(N), N)$, because

$$
e(\{35\}, x)+e(\{124\}, x)=0 \text { and } e(\{35\}, x)=e(\{124\}, x) .
$$

Suppose that, for a certain $\mathscr{D}(\{14\})$, player 1 is separated out, then, for $x \in X_{2}\left(\mathscr{D}(\{14\},\{14\}), x_{1}=0\right.$ and therefore the coalition $\{1\}$ belongs to $\mathscr{D}(N, x)$ (see (4.2)). But, since $x_{4}>0$ for payoff vectors in $\mathscr{K}$, it follows that for such payoff vectors player 4 is not separated out in $\mathscr{D}(N, x)$, and therefore players 1 and 4 separate each other in $\mathscr{D}(N, x)$. Consequently, when computing the second stage, we need not worry about player 1 being separated out. Whether $x_{1}$ is equal to zero or not for a payoff vector in $\mathscr{K}$, this payoff vector must be contained in $X_{2}(\mathscr{D}(\{14\},\{14\})$ for some $\mathscr{D}(\{14\})$ in which players 1 and 4 separate each other.

Because of the saturation conditions, the results of $\S 7$ and the remarks which precede Example 10.6, it is enough to let $\vartheta\left(q_{2}\right)$ contain only the following sets:

$$
\begin{array}{lll}
\mathscr{D}_{2} \equiv\{\{4\},\{1\}\}, & \mathscr{D}_{3} \equiv\{\{4\},\{125\},\{135\}\}, & \mathscr{D}_{4} \equiv\{\{4\},\{123\}\}, \\
\mathscr{D}_{5} \equiv\{\{45\},\{1\}\}, & \mathscr{D}_{6} \equiv\{\{45\},\{125\},\{135\}\}, & \mathscr{D}_{7} \equiv\{\{45\},\{123\}\}, \\
\mathscr{D}_{8} \equiv\{\{234\},\{1\}\}, & \mathscr{D}_{9} \equiv\{\{234\},\{125\},\{135\}\}, & \mathscr{D}_{10} \equiv\{\{234\},\{123\}\},
\end{array}
$$

If $\{4\} \in \mathscr{D}(\{14\})$, then $x_{1}=x_{4}$ for $x \in X_{2}(\mathscr{D}(\{14\}),\{14\})$, because $x_{1}<x_{4}$ implies $s_{1,4}(x)>s_{4,1}(x)$. The only payoff vector $x$ which may perhaps result is $(1 / 3,1 / 3,1 / 3,1 / 3,2 / 3)$, which is not in $X_{2}(\mathscr{D}(\{14\}),\{14\})$, because $-1 / 3=e(\{4\}, x)<e(\{45\}, x)=0$. Thus, $\mathscr{D}(\{14\})$ can only be one of the $\mathscr{D}_{i}^{\prime} \mathrm{s}, i=5,6, \cdots, 10$. A straightforward computation shows that

$$
\begin{aligned}
& \quad X_{2}\left(\mathscr{D}_{1}, N\right) \cap X_{2}\left(\mathscr{D}_{5},\{14\}\right)=X_{2}\left(\mathscr{D}_{1}, N\right) \cap X_{2}\left(\mathscr{D}_{6},\{14\}\right) \\
& \quad=[(0,1 / 2,1 / 2,1 / 2,1 / 2)(1 / 24,11 / 24,11 / 24,12 / 24,13 / 24)] \equiv I_{1}, \\
& X_{2}\left(\mathscr{D}_{1}, N\right) \cap X_{2}\left(\mathscr{D}_{7},\{14\}\right) \\
& \quad=[(7 / 24,8 / 24,8 / 24,9 / 24,16 / 24)(1 / 24,11 / 24,11 / 24,12 / 24,13 / 24)] \equiv I_{2}, \\
& X_{2}\left(\mathscr{D}_{1}, N\right) \cap X_{2}\left(\mathscr{D}_{8},\{14\}\right)=X_{2}\left(\mathscr{D}_{1}, N\right) \cap X_{2}\left(\mathscr{D}_{9},\{14\}\right)=\varnothing, \\
& X_{2}\left(\mathscr{D}_{1}, N\right)^{\prime} \cap X_{2}\left(\mathscr{D}_{10},\{14\}\right) \\
& \quad=[(7 / 24,8 / 24,8 / 24,9 / 24,16 / 24)(11 / 36,11 / 36,11 / 36,14 / 36,25 / 36)]=I_{3},
\end{aligned}
$$

and therefore $\mathscr{K}=I_{1} \cup I_{2} \cup I_{3}$ is a union of three straight line segments. 
11. Favorablness relations. If many desirability relations occur in a game, Theorem 10.4 yields a significant reduction in the representation formula. In general, however, even if a complete order of desirability of players exists, the methods described here do not filter out all the superfiuous polyhedra, and we know of no general method which does. We can suggest, however, another method which may be quite powerful even if no desirability relations exist at all. This will now be explained.

Lemma 11.1. Let $T_{j}^{r} \in q_{r}$ and let $\delta_{r}^{0}, \hat{o}_{r}^{0} \in \vartheta\left(q_{r}\right)$, have the set $\mathscr{D}\left(T_{j}^{r}\right)$ as its $j$-th component. Let

$$
\begin{aligned}
& X_{3}\left(\mathscr{D}\left(T_{j}^{r}\right),\left(T_{j}^{r}\right)\right. \\
& \quad=\left\{x: x \in X(N ; \Xi), e(A, x)=\operatorname{Max}_{s \in \mathscr{c}\left(T_{j}^{r}\right)} e(S)=s\left(T_{j}^{r}, x\right)\right. \\
& \left.\qquad \text { for all } A \in \mathscr{D}\left(T_{j}^{r}\right)\right\} .
\end{aligned}
$$

If there exists a coalition $B$ in $\mathscr{C}\left(T_{j}^{r}\right)$, which does not belong to $\mathscr{D}\left(T_{j}^{r}\right)$, such that $e(B, x)=s\left(T_{j}^{r}, x\right)$ for each $x$ in $X_{3}\left(\mathscr{D}\left(T_{j}^{r}\right), T_{j}^{r}\right)$, then one can remove $\delta_{r}^{0}$ from $^{23} \vartheta\left(q_{r}\right)$ and Theorem 10.4 still holds.

Proof. Clearly, if $\delta_{r}^{0}$ is removed, the right hand side of (4.14) is contained in $\mathscr{K}$. To prove the other inclusion, let $x$ be a payoff vector in $\mathscr{C}$ and $P^{*}(x)$ be the profile constructed in the proof of Theorem 10.4. Clearly, if $\mathscr{D}\left(T_{j}^{r}, x\right)$ contains $\mathscr{D}\left(T_{j}^{r}\right)$, then it also contains the coalition $B$; hence $x$ is determined by the sequence $\delta_{1}^{*}(x), \cdots, \delta_{n \rightarrow 1}^{*}(x)$, which satisfies the requirements of the theorem.

Definition 11.2. Let $T$ be a subset of $N$ and let $\mathscr{D}(T)$ be a collection of coalitions of the class $\mathscr{C}(T)$ in a game $(N ; v)$. A player $k$ is said to be more favorable than a player $l$ with respect to $\mathscr{D}(T)$ and $T$, if $k, l \in T$ and there exists a coalition $A$ in $\mathscr{D}(T)$ which contains player $l$ and does not contain player $k$, such that

$$
v(A) \leqq v((A \cup\{k\})-\{l\}) .
$$

Such a favorableness relation will be denoted by ${ }^{24} \varepsilon_{z(T)} l$. Note that the "weak" player $l$ is put here in a nonsymmetric role with respect to $\mathscr{D}(T)$.

Lemma 11.3. Let $x$ be a payoff vector in a game $(N ; v)$, and let $\mathscr{D}(T, x)$ be the set of those coalitions among the coalitions of $\mathscr{C}(T)$, which attain maximum excess $s(T, x)$ with respect to $x$. If $k \Sigma_{\Sigma(T, x)} l$ then $x_{k} \geqq x_{l}$.

${ }^{23}$ And such a removal may of course reduce the sets $q_{r+1}, 9\left(q_{r+1}\right), \cdots, q_{n-1}$, $\vartheta\left(q_{n-1}\right), q_{n}$.

${ }_{24}$ We are only interested in the relation $\varepsilon_{\mathscr{D}(T, x)} \cdot \mathcal{E}_{\mathscr{X}(T, x)}$ plays no role here. 
Proof. ${ }^{25}$ Suppose $x_{l}>x_{k}$. There exists a coalition $A$ in $\mathscr{D}(T, x)$, $l \in A, k \notin A$, for which (11.2) holds. Clearly, $(A \cup\{k\})-\{l\} \in \mathscr{C}(T)$. By (11.2), $e((A \cup\{k\})-\{l\}, x)-e(A, x) \geqq x_{l}-x_{k}>0$, contrary to the maximality of $e(A, x)$.

REMARK 11.4. In view of the previous lemma, we extend from now on the definition of favorableness with respect to $\mathscr{D}(T)$ by transitive closure. Similarly, because of Theorem 9.3, and since we are only interested in payoff vectors of the kernel, we can combine the desirability and favorableness relations with respect to a $\mathscr{D}(T)$ and close them by transitivity. We shall refer to such an operation as a combined desirability-favorableness relation.

Definition 11.5. Let $T$ be a subset of $N$, and let $\mathscr{D}(T)$ be a set of coalitions of the class $\mathscr{C}(T)$ in a game $(N ; v)$. We say that a coalition $A$ is more prosperous than a coalition $B$, with respect to $\mathscr{D}(T)$, if $A, B \in \mathscr{C}(T)$, if $v(B) \geqq v(A)$ and if, in addition, there exists a one-to-one mapping $\lambda$ of a subset $A_{1}$ of $A$ onto $B$, such that either $i \S_{\mathscr{Q}(T)} \lambda(i)$ or $i \equiv \lambda(i), i \in A_{1}$ (compare Definition 9.8). Such a relation will be denoted by $A \geqq \mathscr{\mathscr { X } ( T )} B$.

Lemma 11.6. If $x \in \mathscr{K}$, if $A \in \mathscr{D}(T, x)$ and $A \geqq \mathscr{\mathscr { S } ( T ^ { \prime } , x )} B$, then $B \in \mathscr{D}(T, x)$ and $x_{i}=0$ for $i \in A-A_{1}$ Here $\mathscr{D}(T, x)$ is defined as in Lemma 11.3 and $A_{1}$ is described in Definition 11.5.

Proof. $e(A, x) \geqq e(B, x)$ because $A, B \in \mathscr{C}(T)$ and $A \in \mathscr{D}(T, x)$. By Lemma 11.3 and Theorem 9.3

$$
e(B, x)=v(B)-\sum_{B} x_{i} \geqq v(A)-\sum_{A_{1}} x_{i}=e(A, x)
$$

and the rest of the proof is straightforward.

By Lemma 11.6, we can impose another type of a saturation condition on the sets $\mathscr{D}\left(T_{j}^{r}\right)$; namely, require in Theorem 10.4 that, in addition to the other requirements, if $A \in \mathscr{D}\left(T_{j}^{r}\right)$ and $B \in \mathscr{C}\left(T_{j}^{r}\right)$, and if $A \geqq \mathscr{S}_{\left(T_{j}^{r}\right)} B$, then $B \in \mathscr{D}\left(T_{j}^{r}\right)$. The remarks which follow Example 10.5 are valid also in this case.

EXAMPLE 11.7. Consider the 5-person game $(N ; v)$, where $v(S)=1$ for $S \in\{\{12\},\{24\},\{34\},\{23\},\{14\},\{35\},\{25\},\{12345\}\}$, and $v(S)=0$ otherwise. Its desirability relations are $1 \geq 2,3,4,3 \approx 2,4 \geq 2,532,3,4$. The following table can easily be computed.

${ }^{25}$ Note that unlike the case of Theorem 9.3 , we do not require $x$ to belong to the kernel. 


\begin{tabular}{|c|c|c|}
\hline $\begin{array}{l}\text { If } S \text { is in } \\
\mathscr{D}(N) \\
\quad S\end{array}$ & $\begin{array}{c}\text { then the } T^{\prime} s \text { are also } \\
\text { in } \mathscr{D}(N) \\
T\end{array}$ & $\begin{array}{c}\text { and the combined desirability } \\
\text { favorableness relations are } \\
\varepsilon_{\mathscr{g}}\end{array}$ \\
\hline$\{12\}$ & $\{14\}$ & $1 \xi_{\mathscr{D}} 52=4$ \\
\hline$\{24\}$ & $\{14\},\{34\},\{12\},\{25\},\{35\}$ & $1={ }_{\mathscr{\partial}} 2=_{\mathscr{\partial}} 3={ }_{\mathscr{O}} 4=_{\mathscr{D}} 5$ \\
\hline$\{34\}$ & $\{35\},\{14\}$ & $1=\mathscr{D}^{3} \quad 4=\mathscr{D}^{5}$ \\
\hline$\{23\}$ & $\{34\},\{35\},\{14\},\{12\},\{25\}$ & 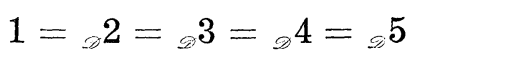 \\
\hline$\{14\}$ & - & 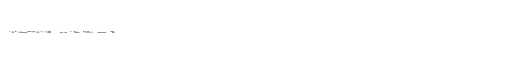 \\
\hline$\{35\}$ & - & (n) \\
\hline$\{25\}$ & $\{35\}$ & $2=3 \quad 5 \varepsilon_{\mathscr{D}} 1$ \\
\hline
\end{tabular}

Looking at the various candidates for $\mathscr{D}(N)$, we can somewhat shorten the computations by the following observations:

$a$. For $x \in \mathscr{K}$, it follows from the desirability relations and from Theorem 9.3 that $x_{2}>0$. Therefore, only such $\mathscr{D}(N)^{\prime}$ 's need be considered, for which player 2 is not separated out.

$b$. For $x \in \mathscr{K}, x_{3}+x_{4}>0$. Indeed, otherwise, player 3 will outweigh any player who receives a positive payoff. Therefore, only such $\mathscr{D}(N)^{\prime}$ 's need be considered for which either player 3 or player 4 is not separated out.

In order to satisfy $a$. and $b ., \mathscr{D}(N)$ must contain at least five coalitions. A glance in the secend column of the table shows that any such set must contain coalitions $\{12\},\{25\}$ and $\{34\}$, which implies that all the players must be equal under the combined desirabilityfavorableness relations. Thus, all the 2-person winning coalitions must be in $\mathscr{D}(N)$ and $(1 / 5,1 / 5,1 / 5,1 / 5,1 / 5)$ is the unique point in the kernel.

The same ideas can further be generalized by introducing favorableness relations among sets of players. We shall state the definitions and the lemmas, but the proofs will be omitted; they resemble the proofs of Lemmas 11.3 and 11.6.

Definition 11.8. Let $T$ be a subset of $N$, and let $\mathscr{D}(T)$ be a set of coalitions of the class $\mathscr{C}(T)$ in a game $(N ; v)$. A set of players $\left\{k_{1}, k_{2}, \cdots, k_{\alpha}\right\}$ is said to be more favorable than a set of players $\left\{l_{1}, l_{2}, \cdots, l_{\beta}\right\}$, with respect to $\mathscr{D}(T)$, if $k_{1}, \cdots, k_{\alpha}, l_{1}, \cdots, l_{\beta} \in T$, and if there exists a coalition $A$ in $\mathscr{D}(T)$ which contains the second set and does not intersect the first set, such that

$$
v(A) \leqq v\left(\left(A \cup\left\{k_{1}, \cdots, k_{\alpha}\right\}\right)-\left\{l_{1}, \cdots, l_{\beta}\right\}\right) .
$$


Moreover, this definition is further extended by transitive closure ${ }^{26}$. We shall write $\left\{k_{1}, \cdots, k_{\alpha}\right\} \S_{\mathscr{D}(\boldsymbol{T})}\left\{l_{1}, \cdots, l_{\beta}\right\}$.

LEMma 11.9. Let $x$ be a payoff vector in a game $(N ; v)$ and let $\mathscr{D}(T, x)$ be the set of coalitions among the coalitions of $\mathscr{C}(T)$, which attain maximum excess $s(T, x)$ with respect to $x$. If $\left\{k_{1}, \cdots, k_{\alpha}\right\} \Xi_{\mathscr{T}(T, x)}\left\{l_{1}, \cdots, l_{\beta}\right\}$ then $x_{k_{1}}+\cdots+x_{k_{\alpha}} \geqq x_{l_{1}}+\cdots+x_{l_{\beta}}$.

Definition 11.10. Let $T$ be a subset of $N$, and let $\mathscr{D}(T)$ be a set of coalitions of the class $\mathscr{C}(T)$ in a game $(N ; v)$. We say that a coalition $A$ is more prosperous than a coalition $B$, with respect to $\mathscr{D}(T)$, if $A, B \in \mathscr{C}(T)$, if $v(B) \geqq v(A)$ and if, in addition, there exists a partition $\left\{A_{11}, A_{12}, \cdots, A_{1 t}\right\}$ of a subcoalition $A_{1}, A_{1} \subset A$, and a partition $\left\{B_{1}, B_{2}, \cdots, B_{t}\right\}$ of $B$, such that either $A_{1 \nu} \cong B_{\nu}$ or $A_{1 \nu} \varepsilon_{\mathscr{D}(T)} B_{\nu}$, $\nu=1,2, \cdots, t$. We write $A \geqq{ }_{\mathscr{g}(\boldsymbol{T})} B$.

Lemma 11.11. If $x \in X(N$; $\leftleftarrows)$, if $A \in \mathscr{D}(T, x)$ and $A \geqq{ }_{\mathscr{D}\left(T^{r}, x\right)} B$, then $B \in \mathscr{D}(T, x)$ and $x_{i}=0$ for $i \in A-A_{1}$. Here, $\mathscr{D}(T, x)$ is defined as in Lemma 11.3 and $A_{1}$ is described in Definition 11.10.

Thus, in addition to the saturation condition, involved in Theorem 10.4 , on the sets $\mathscr{D}\left(T_{j}^{r}\right)$ one can require that if $A \in \mathscr{D}\left(T_{j}^{r}\right), B \in \mathscr{C}\left(T_{j}^{r}\right)$ and $A \geqq{ }_{\mathscr{g}\left(r_{j}^{r}\right)}^{r} B$, then $B \in \mathscr{D}\left(T_{j}^{r}\right)$. The remarks which follow Example 10.5 are valid also here.

\section{REFERENCES}

1. R. J. Aumann and M. Maschler, The bargaining set for cooperative games, Advances in Game Theory, M. Dresher, L. S. Shapley and A. W. Tucker, eds., Annals of Mathematics Studies, No. 52, Princeton University Press, Princeton, 1964, 443-476. 2. R. J. Aumann, B. Peleg and P. Rabinowitz, A method for computing the kernel of n-person games, Mathematics of Computation, 19 (1965), 531-551.

3. M. Davis and M. Maschler, Existence of stable payoff configurations for cooperative games, Bull. Amer. Math. Soc. 69 (1963), 106-108. A detailed paper with the same title will appear in Studies in Mathematical Economics, Essays in Honor of Oskar Morgenstern, M. Shubik, ed.

4. 느, The kernel of a cooperative game, (to appear in Naval Research Logistics Quarterly.)

5. D. Gale, The Theory of linear economic models, McGraw-Hill Book Company, New York, 1960.

6. J. R. Isbell, A class of simple games, Duke Math. J. 25 (1958), 423-439.

7. B. Peleg, The kernel of constant-sum simple games with homogeneous weights, (to appear in Illinois J. Math).

8. ㄴ The kernel of m-quota games, Canadian J. Math. 17 (1965), 239-244.

9. - Existence theorem for the bargaining set $\mathscr{M}_{1}^{(i)}$, Bull. Amer. Math. Soc. 69 (1963), 109-110. A detailed paper with the same title will appear in Studies in Mathematical Economics, Essays in Honor of O. Morgenstern, M. Shubik, ed.

${ }^{26}$ See Remark 11.4. 
10. L. S. Shapley, Simple games; an outline of the descriptive theory, Behavioral Science 7 (1962), 59-66.

11. - A value for n-person games, Contributions to the Theory of Games, Vol. II, Annals of Mathematics Studies No. 28, H. W. Kuhn and A. W. Tucker, eds., Princeton University Press, Princeton 1953, 307-317.

12. L. S. Shapley and M. Shubik, The core of an economy with nonconvex preferences, The RAND Corporation, Santa Monica, California, RM-3518-PR, Feb. 1963.

13. J. Von Neumann and O. Morgenstern, Theory of games and economic behavior, Princeton University press, 1944.

The Hebrew University

JERUSALEM, ISRAEL 


\section{PACIFIC JOURNAL OF MATHEMATICS}

\section{EDITORS}

H. SAMELSON

Stanford University

Stanford, California

R. M. BLUMENTHAL

University of Washington

Seattle, Washington 98105

\section{*J. DugundJI}

University of Southern California Los Angeles, California 90007

RICHARD ARENS

University of California

Los Angeles, California 90024

\section{ASSOCIATE EDITORS}

E. F. BECKENBACH
F. WOLF

K. YosIDA

\section{SUPPORTING INSTITUTIONS}

\author{
UNIVERSITY OF BRITISH COLUMBIA \\ CALIFORNIA INSTITUTE OF TECHNOLOGY \\ UNIVERSITY OF CALIFORNIA \\ MONTANA STATE UNIVERSITY \\ UNIVERSITY OF NEVADA \\ NEW MEXICO STATE UNIVERSITY \\ OREGON STATE UNIVERSITY \\ UNIVERSITY OF OREGON \\ OSAKA UNIVERSITY \\ UNIVERSITY OF SOUTHERN CALIFORNIA
}

\author{
STANFORD UNIVERSITY \\ UNIVERSITY OF TOKYO \\ UNIVERSITY OF UTAH \\ WASHINGTON STATE UNIVERSITY \\ UNIVERSITY OF WASHINGTON \\ AMERICAN MATHEMATICAL SOCIETY \\ CHEVRON RESEARCH CORPORATION \\ TRW SYSTEMS \\ NAVAL ORDNANCE TEST STATION
}

Mathematical papers intended for publication in the Pacific Journal of Mathematics should be typewritten (double spaced). The first paragraph or two must be capable of being used separately as a synopsis of the entire paper. It should not contain references to the bibliography. Manuscripts may be sent to any one of the four editors. All other communications to the editors should be addressed to the managing editor, Richard Arens at the University of California, Los Angeles, California 90024 .

50 reprints per author of each article are furnished free of charge; additional copies may be obtained at cost in multiples of 50 .

The Pacific Journal of Mathematics is published monthly. Effective with Volume 16 the price per volume (3 numbers) is $\$ 8.00$; single issues, $\$ 3.00$. Special price for current issues to individual faculty members of supporting institutions and to individual members of the American Mathematical Society: $\$ 4.00$ per volume; single issues $\$ 1.50$. Back numbers are available.

Subscriptions, orders for back numbers, and changes of address should be sent to Pacific Journal of Mathematics, 103 Highland Boulevard, Berkeley 8, California.

Printed at Kokusai Bunken Insatsusha (International Academic Printing Co., Ltd.), No. 6, 2-chome, Fujimi-cho, Chiyoda-ku, Tokyo, Japan.

PUBLISHED BY PACIFIC JOURNAL OF MATHEMATICS, A NON-PROFIT CORPORATION

The Supporting Institutions listed above contribute to the cost of publication of this Journal, but they are not owners or publishers and have no responsibility for its content or policies.

* Paul A. White, Acting Editor until J. Dugundji returns. 


\section{Pacific Journal of Mathematics}

\section{Vol. 18, No. 2 \\ April, 1966}

Alexander V. Arhangelskii, On closed mappings, bicompact spaces, and a problem of P. Aleksandrov .............................. 201

A. K. Austin, A note on loops . . . . . . . . . . . . . . . . . . . . . . . . . . . . 209

Lawrence Peter Belluce and William A. Kirk, Fixed-point theorems for families of contraction mappings...................... 213

Luther Elic Claborn, Every abelian group is a class group ............ 219

Luther Elic Claborn, A note on the class group .................. 223

Robert Stephen De Zur, Point-determining homomorphisms on multiplicative semi-groups of continuous functions............. 227

Raymond William Freese, A convexity property ................ 237

Frederick Paul Greenleaf, Characterization of group algebras in terms of their translation operators ......................... 243

Andrzej Hulanicki, On the spectral radius of hermitian elements in group algebras....................................... 277

Michael Bahir Maschler and Bezalel Peleg, A characterization, existence proof and dimension bounds for the kernel of a game ............ 289

Yiannis (John) Nicolas Moschovakis, Many-one degrees of the predicates

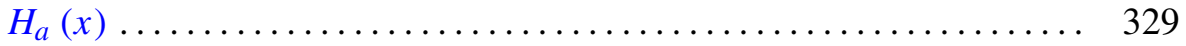

G. O. Okikiolu, $n$th order integral operators associated with Hilbert transforms.

C. E. Rickart, Analytic phenomena in general function algebras ... 361

K. N. Srivastava, On an entire function of an entire function defined by Dirichlet series

Paul Elvis Waltman, Oscillation criteria for third order nonlinear differential equations .......................... 